\title{
Complementary roles of mechanotransduction and inflammation in vascular homeostasis
}

Citation for published version (APA):

Latorre, M., Spronck, B., \& Humphrey, J. D. (2021). Complementary roles of mechanotransduction and inflammation in vascular homeostasis. Proceedings of the Royal Society A-Mathematical Physical and Engineering Sciences, 477(2245), [20200622]. https://doi.org/10.1098/rspa.2020.0622

Document status and date:

Published: 27/01/2021

DOI:

10.1098/rspa.2020.0622

Document Version:

Publisher's PDF, also known as Version of record

\section{Please check the document version of this publication:}

- A submitted manuscript is the version of the article upon submission and before peer-review. There can be important differences between the submitted version and the official published version of record.

People interested in the research are advised to contact the author for the final version of the publication, or visit the DOI to the publisher's website.

- The final author version and the galley proof are versions of the publication after peer review.

- The final published version features the final layout of the paper including the volume, issue and page numbers.

Link to publication

\footnotetext{
General rights rights.

- You may freely distribute the URL identifying the publication in the public portal. please follow below link for the End User Agreement:

www.umlib.nl/taverne-license

Take down policy

If you believe that this document breaches copyright please contact us at:

repository@maastrichtuniversity.nl

providing details and we will investigate your claim.
}

Copyright and moral rights for the publications made accessible in the public portal are retained by the authors and/or other copyright owners and it is a condition of accessing publications that users recognise and abide by the legal requirements associated with these

- Users may download and print one copy of any publication from the public portal for the purpose of private study or research.

- You may not further distribute the material or use it for any profit-making activity or commercial gain

If the publication is distributed under the terms of Article $25 \mathrm{fa}$ of the Dutch Copyright Act, indicated by the "Taverne" license above, 


\section{PROCEEDINGS A}

rspa.royalsocietypublishing.org

\section{Research 8 (}

Article submitted to journal

\section{Subject Areas:}

Biomedical Engineering,

Mathematical Modelling, Computer

Modelling and Simulation

\section{Keywords:}

hypertension, inflammation, homeostasis, mechanotransduction, artery

\section{Author for correspondence:}

Jay D. Humphrey

e-mail: jay.humphrey@yale.edu

\section{THE ROYAL SOCIETY} PUBLISHING

\section{Complementary Roles of Mechanotransduction and Inflammation in Vascular Homeostasis}

Marcos Latorre ${ }^{1}$, Bart Spronck ${ }^{1,2}$ and

Jay D. Humphrey ${ }^{1,3}$

\author{
${ }^{1}$ Department of Biomedical Engineering, Yale \\ University, New Haven, CT, USA \\ ${ }^{2}$ Department of Biomedical Engineering, CARIM \\ School for Cardiovascular Diseases, Maastricht \\ University, Maastricht, The Netherlands \\ ${ }^{3}$ Vascular Biology and Therapeutics Program, Yale \\ School of Medicine, New Haven, CT, USA
}

Arteries are exposed to relentless pulsatile hemodynamic loads, but via mechanical homeostasis they tend to maintain near optimal structure, properties, and function over long periods in maturity in health. Numerous insults can compromise such homeostatic tendencies, however, resulting in maladaptations or disease. Chronic inflammation can be counted among the detrimental insults experienced by arteries, yet inflammation can also play important homeostatic roles. In this paper, we present a new theoretical model of complementary mechanobiological and immunobiological control of vascular geometry and composition, and thus properties and function. We motivate and illustrate the model using data for aortic remodeling in a common mouse model of induced hypertension. Predictions match the available data well, noting a need for increased data for further parameter refinement. The overall approach and conclusions are general, however, and help to unify two previously disparate literatures, thus leading to deeper insight into the separate and overlapping roles of mechanobiology and immunobiology in vascular health and disease.

\section{Introduction}

The concept of homeostasis was introduced by Walter Cannon in the 1920s, extending the notion of a stable

(C) The Authors. Published by the Royal Society under the terms of the Creative Commons Attribution License http://creativecommons.org/licenses/ by $/ 4.0 /$, which permits unrestricted use, provided the original author and source are credited. 
internal environment (mileu intérieur) put forth by Claude Bernard in the 1870s. Over the years, homeostasis has come to be known as a fundamental biological and physiological process by which a select quantity is regulated to remain, within a particular range, near a target value that is often referred to as a set-point. This process is achieved via negative feedback and is thought to promote stable, near optimal function. Two prime examples include regulation of interstitial fluid $\mathrm{pH}$, at a microscale, and regulation of core body temperature, at a macroscale. Although metrics of continuum biomechanics such as mechanical stresses cannot be sensed or regulated directly by cells [1], they have proven useful as easily calculated surrogates as evidenced in the vasculature, for example, by the narrow range of stress calculated at focal adhesions in fibroblasts and smooth muscle cells [2,3] as well as the robust regulation of time averaged blood flow-induced wall shear stress at the endothelial surface [4,5] and regulation of mean blood pressure-induced intramural stress [6,7], both within and across species. Hence, mechanical homeostasis manifests across spatio-temporal scales in the vasculature [8]. Macroscale mechano-regulation of an artery can be achieved over short periods by vasoregulation of caliber, but over long periods must include turnover of extracellular matrix within potentially evolving mechanical states. Such regulation occurs via altered gene expression that can change cell number and phenotype as well as matrix composition and organization, often in direct response to the sustained alterations in hemodynamic stimuli.

Whereas chemical, thermal, and mechanical homeostasis have long been appreciated, roles played by inflammation in homeostasis have been recognized much more recently. It is now clear, however, that resident macrophages can promote tissue homeostasis by clearing apoptotic cells and cellular debris as well as by removing degraded or damaged matrix $[9,10]$. Importantly, like all primary vascular cells - endothelial, smooth muscle, and fibroblasts - macrophages are highly sensitive to changes in their mechanical environment [11,12], thus they too can contribute directly to mechanical homeostasis. Although the immune system evolved to protect against lifethreatening pathological insults, including bacterial and viral, an emerging paradigm suggests that inflammatory processes also engage when normal homeostatic processes are not sufficient to restore conditions when perturbed from normal [13]. The term "para-inflammation" was coined to delineate these supportive homeostatic functions from the primary protective functions of inflammation [14]. Regardless of terminology, the key observation is that inflammatory cells can come to the aid of tissue specific cells to promote homeostasis when the latter are unable to respond sufficiently or quickly enough to recover from a perturbation. One caveat, however, is that inflammatory cells, having priority because of their ability to defend against life-threatening insults, can alter normal homeostatic parameters, including set-points and gains in the negative feedback system, and thereby establish a new homeostatic state, compromise homeostasis altogether, or even drive the tissue toward disease [15]. There is, therefore, a pressing need to understand better both the complementary and contrasting roles of immuno-mechano-regulation of tissue structure and function (Figure 1).

The importance of inflammation in the remodeling of elastic and muscular arteries was demonstrated in 2008 in cases of sustained alterations in flow, with inflammation resolving quickly following the homeostatic response [16,17]. Soon thereafter, it was shown that resident macrophages play a key role in such remodeling [18], though many different types of inflammatory cells can participate, some with layer specificity [19]. In this paper, we present a new theoretical framework for modeling one aspect of mechanical stress-mediated inflammation and its role in vascular homeostasis or its loss. The framework is motivated by prior findings but informed directly by recent data on aortic remodeling collected in our laboratory for a common mouse model of induced hypertension, which elevates blood pressure above normal values and thereby perturbs intramural stresses from original set-points. We illustrate the utility of the model by computing evolving changes in wall geometry, composition, and properties, emphasizing for the first time the important consequences not only of inflammatory support versus supremacy but also of consequences of smooth muscle cell phenotypic modulation. As it can be seen, the model can capture and delineate effects of different degrees of inflammation as the hypertensive 
aorta either adapts or maladapts in response to a sustained elevation in blood pressure that is driven by a pro-inflammatory mediator.

\section{Methods}

\section{(a) Computational model}

We recently showed that a computational model of arterial growth and remodeling (G\&R) that includes mechano- and immuno-stimulated matrix turnover can capture salient biomechanical features of the time-course of maladaptive remodeling of the thoracic aorta in both C57BL/ 6 and $A p o e^{-/-}$(on a C57BL/6 background) mice infused with angiotensin II (AngII) for a period of weeks $[20,21]$. Briefly, this constrained mixture model allows one to account for the evolution of mass fractions, mechanical properties, deposition stretches, and rates of turnover of multiple structurally significant constituents. Among the key equations are the mixture relation for the strain energy function per unit reference volume

$$
W_{\Gamma \mathrm{R}}^{\alpha}(s)=\frac{1}{\rho} \int_{-\infty}^{s} m_{\Gamma \mathrm{R}}^{\alpha}(\tau) q_{\Gamma}^{\alpha}(s, \tau) \hat{W}_{\Gamma}^{\alpha}\left(\mathbf{C}_{\Gamma \mathrm{n}(\tau)}^{\alpha}(s)\right) d \tau
$$

where $\rho$ is the wall mass density, $m_{\Gamma \mathrm{R}}^{\alpha}(\tau)>0$ denotes the true rate of mass density production per unit reference volume at $\operatorname{G\& R}$ time $\tau, q_{\Gamma}^{\alpha}(s, \tau) \in[0,1]$ is the fraction of material produced at time $\tau$ that survives to the current time $s \geq \tau$, and $\hat{W}^{\alpha}$ is the stored energy function; each term is constituent- ( $\alpha=1,2, \ldots, N$, accounting mainly for the dominant structural constituents, namely, elastic fibers, smooth muscle, and multiple families of collagen fibers) and layer- $(\Gamma=$ $M, A$ for media and adventitia, respectively) specific. $\mathbf{C}_{\Gamma \mathrm{n}(\tau)}^{\alpha}(s)=\mathbf{F}_{\Gamma \mathrm{n}(\tau)}^{\alpha \mathrm{T}}(s) \mathbf{F}_{\Gamma \mathrm{n}(\tau)}^{\alpha}(s)$ is the right Cauchy-Green tensor where

$$
\mathbf{F}_{\Gamma \mathrm{n}(\tau)}^{\alpha}(s)=\mathbf{F}_{\Gamma}(s) \mathbf{F}_{\Gamma}^{-1}(\tau) \mathbf{G}_{\Gamma}^{\alpha}(\tau)
$$

is the constituent- and layer-specific deformation gradient, with $\mathrm{n}(\tau)$ denoting potentially evolving constituent-specific natural (stress-free) configurations, $\mathbf{F}_{\Gamma}$ capturing mixture-level deformations (at G\&R time $s$ or $\tau$ ) relative to a common reference configuration, and $\mathbf{G}_{\Gamma}^{\alpha}(\tau)$ representing the "deposition stretch" at which the constituent is incorporated within each layer. Based on prior successes in modeling vascular G\&R, let the mass density production and survival functions be governed constitutively by

$$
m_{\Gamma \mathrm{R}}^{\alpha}(\tau)=k_{\Gamma}^{\alpha}(\tau) \rho_{\Gamma \mathrm{R}}^{\alpha}(\tau) \Upsilon_{\Gamma}^{\alpha}(\tau)
$$

and

$$
q_{\Gamma}^{\alpha}(s, \tau)=\exp \left(-\int_{\tau}^{s} k_{\Gamma}^{\alpha}(t) d t\right)
$$

where $k_{\Gamma}^{\alpha}>0$ is a rate parameter that governs constituent removal via a first-order type of kinetic decay, $\rho_{\Gamma \mathrm{R}}^{\alpha}$ is the associated referential mass density, and $\Upsilon_{\Gamma}^{\alpha}>0$ is a function that stimulates mass production at $\left(\Upsilon_{\Gamma}^{\alpha}=1\right)$, below $\left(\Upsilon_{\Gamma}^{\alpha}<1\right)$, or above $\left(\Upsilon_{\Gamma}^{\alpha}>1\right)$ basal levels. The (convolution integral for) mass density evolution reads

$$
\rho_{\Gamma \mathrm{R}}^{\alpha}(s)=\int_{-\infty}^{s} m_{\Gamma \mathrm{R}}^{\alpha}(\tau) q_{\Gamma}^{\alpha}(s, \tau) d \tau .
$$

Further constitutive assumptions include a stress-dependent rate parameter for constituent removal, assumed to take the form

$$
k_{\Gamma}^{\alpha}(t)=k_{\Gamma 0}^{\alpha}\left(1+(\Delta \sigma(t))^{2}\right)
$$

where $k_{\Gamma 0}^{\alpha}$ denotes a basal rate of removal (noting that both increases and decreases in stress relative to its homeostatic set-point can hasten constituent removal, modeled phenomenologically here given our current imprecise understanding of the complexities associated with stress affecting rates of protease production and activation as well as affecting the degree of 
vulnerability of the matrix to the protease) as well as an immuno-mechano-stimulus function for constituent production of the form

$$
\Upsilon_{\Gamma}^{\alpha}(\tau)=1+f_{\Gamma \sigma}^{\alpha}(\Delta \sigma(\tau))-f_{\Gamma \tau_{w}}^{\alpha}\left(\Delta \tau_{w}(\tau)\right)+f_{\Gamma \varrho_{\varphi}}^{\alpha}\left(\Delta \varrho_{\varphi}(\tau)\right)
$$

where $\Delta \sigma=\left(\sigma-\sigma_{o}\right) / \sigma_{o}$ and $\Delta \tau_{w}=\left(\tau_{w}-\tau_{w o}\right) / \tau_{w o}$ are normalized deviations in pressure- and axial force-induced intramural stress $\sigma$ and flow-induced wall shear stress $\tau_{w}$ from homeostatic values ( $\sigma_{o}$ and $\tau_{w o}$, respectively, each scalar metrics), and $\Delta \varrho_{\varphi}=\rho_{\mathrm{R} \varphi} / \rho_{\mathrm{R} \varphi \max } \in[0,1]$ is an inflammatory cell fraction relative to its maximum possible referential density $\rho_{\mathrm{R} \varphi \max }$ (cf. Figure 3 in [22]), with $f_{\Gamma \sigma}^{\alpha}, f_{\Gamma \tau_{w}}^{\alpha}$ and $f_{\Gamma \varrho_{\varphi}}^{\alpha}$ generally nonlinear, monotonically increasing functions such that $f_{\Gamma \eta}^{\alpha}(0)=0$ for $\eta=\sigma, \tau_{w}, \varrho_{\varphi}$, with linear approximations performing well under modest perturbations. Importantly, these three quantities are wall $\left(\Delta \sigma\right.$ and $\left.\Delta \varrho_{\varphi}\right)$ or luminal $\left(\Delta \tau_{w}\right)$ averages, with the constituent- and layer-specific functions $f_{\Gamma \eta}^{\alpha}$ (or their gain-type parameters, if linearized) modulating respective changes in cell/matrix production rate within each layer.

Also following our prior study [20], let the intramural elastic fibers, smooth muscle, and collagen fibers be described by the following stored energy functions:

$$
\hat{W}^{\alpha}\left(\mathbf{C}_{\Gamma}^{\alpha}(s)\right)=\frac{c^{\alpha}}{2}\left(\mathbf{C}_{\Gamma}^{\alpha}(s): \mathbf{I}-3\right)
$$

for an amorphous elastin-dominated matrix $(\alpha=e)$, where $c^{e}$ is a shear modulus, and

$$
\hat{W}^{\alpha}\left(\lambda_{\mathrm{n}(\tau)}^{\alpha}(s)\right)=\frac{c_{1}^{\alpha}}{4 c_{2}^{\alpha}}\left[e^{c_{2}^{\alpha}\left(\left(\lambda_{\mathrm{n}(\tau)}^{\alpha}(s)\right)^{2}-1\right)^{2}}-1\right]
$$

for a circumferentially oriented composite of collagen fibers and passive smooth muscle $(\alpha=m)$ plus axially and diagonally oriented collagen fibers $(\alpha=c)$ in the media as well as circumferentially, axially, and diagonally oriented collagen fibers in the adventitia, with $c_{1}^{\alpha}\left(\Delta \varrho_{\varphi}\right)$ and $c_{2}^{\alpha}(\Delta \varrho \varphi)$ possibly inflammation-dependent material parameters (noting that inflammation may override homeostatic set-points, alter the turnover of these constituents by altering gains for production and rates of removal, and modify the mechanical properties of the newly produced constituents) and $\lambda_{n(\tau)}^{\alpha}(s)$ the corresponding stretch. Together, these functions constitute a layerspecific "four-fiber family" model, with effects of other constituents (such as proteoglycans) and cross-links captured phenomenologically via a fit to data. It can be shown that the Cauchy stress

$$
\boldsymbol{\sigma}_{\Gamma}^{\alpha}(s)=\frac{1}{\rho} \int_{-\infty}^{s} m_{\Gamma}^{\alpha}(\tau) q_{\Gamma}^{\alpha}(s, \tau) \hat{\boldsymbol{\sigma}}_{\Gamma}^{\alpha}(s, \tau) d \tau
$$

derives from the stored energy in Eq. (2.1), with

$$
\hat{\boldsymbol{\sigma}}_{\Gamma}^{\alpha}(s, \tau)=\frac{2}{J_{\Gamma \mathrm{n}(\tau)}^{\alpha}(s)} \mathbf{F}_{\Gamma \mathrm{n}(\tau)}^{\alpha}(s) \frac{\partial \hat{W}^{\alpha}\left(\mathbf{C}_{\Gamma \mathrm{n}(\tau)}^{\alpha}(s)\right)}{\partial \mathbf{C}_{\Gamma \mathrm{n}(\tau)}^{\alpha}(s)} \mathbf{F}_{\Gamma \mathrm{n}(\tau)}^{\alpha \mathrm{T}}(s)
$$

where $m_{\Gamma}^{\alpha}(\tau)=m_{\Gamma \mathrm{R}}^{\alpha}(\tau) / J_{\Gamma}(\tau)$ and $J_{\Gamma \mathrm{n}(\tau)}^{\alpha}(s)=\operatorname{det} \mathbf{F}_{\Gamma \mathrm{n}(\tau)}^{\alpha}(s)=J_{\Gamma}(s) / J_{\Gamma}(\tau)$, with $J_{\Gamma}=$ $\operatorname{det} \mathbf{F}_{\Gamma}=\sum \rho_{\Gamma \mathrm{R}}^{\alpha} / \rho$. Finally, consider an active stress contribution in the medial layer in the circumferential direction [23]

$$
\sigma_{M \theta \theta}^{\mathrm{act}}(s)=\phi_{M}^{m}(s) T_{\max }\left(1-e^{-\left(C_{\mathrm{B}}-C_{\mathrm{S}} \Delta \tau_{w}(s)\right)^{2}}\right) \lambda_{\theta, \text { act }}(s)\left(1-\left(\frac{\lambda_{\mathrm{M}}-\lambda_{\theta, \text { act }}(s)}{\lambda_{\mathrm{M}}-\lambda_{0}}\right)^{2}\right)
$$

where $\phi_{M}^{m}=\rho_{M}^{m} / \rho$ is the spatial mass fraction, $\lambda_{\theta \text {, act }}$ the active circumferential stretch, $T_{\max }$ the basal tone, $\lambda_{\mathrm{M}}$ and $\lambda_{0}$ levels of stretch at which contraction is maximal or minimal, and $C_{\mathrm{B}}$ and $C_{\mathrm{S}}$ vasoactive parameters that regulate the contractile response via the flow-induced wall shear stress. The circumferential stretch $\lambda_{\theta \text {, act }}(s)=a(s) / a_{\text {act }}(s)$, with $a(s)$ the current luminal radius and $a_{\text {act }}(s)$ an active reference length that describes the shift in vasomotor tone via rearrangement 
of smooth muscle cells observed in mature arteries via [23,24]

$$
a_{\text {act }}(s)=\int_{-\infty}^{s} k_{\text {act }} a(t) e^{-k_{\text {act }}(s-t)} d t
$$

with $k_{\text {act }}$ the associated rate parameter. In particular, $a_{\text {act }}(0)=a(0)$ (i.e., $\left.\lambda_{\theta \text {,act }}(0)=1\right)$ and $a_{\text {act }}(s \gg 0) \rightarrow a(s \gg 0)$ (i.e., $\left.\lambda_{\theta \text {,act }}(s \gg 0) \rightarrow 1\right)$ when active remodeling is complete. Additional details regarding the G\&R model development and implementation can be found elsewhere [20]

\section{(b) Stress-mediated inflammation}

The convolution integral-based framework has proven useful because the mechanical contributions and rates of removal of some constituents can depend on the time at which they were incorporated within the extant matrix. Nevertheless, rate-based formulations offer advantages in other situations, as, for example, when examining mechanobiological stability $[25,26]$. Here, it proves useful constitutively to consider a rate-based approach. Differentiation of Eq. (2.5) with respect to G\&R time $s$ yields the mass balance relation for constituent $\alpha$ written per unit reference volume

$$
\dot{\rho}_{\Gamma \mathrm{R}}^{\alpha}(s)=m_{\Gamma \mathrm{R}}^{\alpha}(s)-k_{\Gamma}^{\alpha}(s) \rho_{\Gamma \mathrm{R}}^{\alpha}(s)=m_{\Gamma \mathrm{R}}^{\alpha}(s)-n_{\Gamma \mathrm{R}}^{\alpha}(s)
$$

which states that the rate of change of referential mass density $\left(\dot{\rho}_{\Gamma \mathrm{R}}^{\alpha}\right)$ is given by the $(\mathrm{im})$ balance between its true rate of mass density production $\left(m_{\Gamma \mathrm{R}}^{\alpha}>0\right)$ and rate of removal $\left(n_{\Gamma \mathrm{R}}^{\alpha}=k_{\Gamma}^{\alpha} \rho_{\Gamma \mathrm{R}}^{\alpha}>\right.$ 0). Assuming Eq. (2.3) for production yields $\dot{\rho}_{\Gamma \mathrm{R}}^{\alpha}=k_{\Gamma}^{\alpha} \rho_{\Gamma \mathrm{R}}^{\alpha}\left(\Upsilon_{\Gamma}^{\alpha}-1\right)$, which is an evolution equation in rate form (cf. Eq. (2.5) in integral form) that describes well the mass turnover of load-bearing constituents within the arterial wall via the immuno-mechano-mediated stimulus function $\Upsilon_{\Gamma}^{\alpha}$ in Eq. (2.7).

The wall stress-mediated evolution of inflammatory cell density may be described by an evolution equation (or mass balance relation per unit reference volume), analogous to Eq. (2.14),

$$
\dot{\rho}_{\mathrm{R} \varphi}(s)=m_{\mathrm{R} \varphi}(s)-n_{\mathrm{R} \varphi}(s)
$$

aimed to capture the infiltration/activation or loss of inflammatory cells. In particular, we assume that there are no active inflammatory cells at the onset of hypertension $\left(\rho_{\mathrm{R} \varphi}(s=0)=0\right.$; cf. [22]). Once present, subsequent removal is again described by first order kinetics, hence the rate of loss is given by $n_{\mathrm{R} \varphi}=k_{\varphi} \rho_{\mathrm{R} \varphi}$ with $k_{\varphi}$ an associated rate parameter (cf. [27]). With a normalized rate of infiltration/activation $\mu_{\varphi}=m_{\mathrm{R} \varphi} / \rho_{\mathrm{R} \varphi \max }$, yet to be prescribed constitutively, Eq. (2.15) reads

$$
\Delta \dot{\varrho}_{\varphi}(s)=\mu_{\varphi}(s)-k_{\varphi} \Delta \varrho_{\varphi}(s)
$$

which, considering the initial condition $\Delta \varrho_{\varphi}(s=0)=0$, admits a similar (convolution) solution as for the load-bearing constituents (cf. Eq. (2.5))

$$
\Delta \varrho_{\varphi}(s)=\int_{0}^{s} \mu_{\varphi}(\tau) q_{\varphi}(s, \tau) d \tau
$$

with a survival function $q_{\varphi}(s, \tau)=\exp \left(-k_{\varphi}(s-\tau)\right) \forall \tau \in[0, s]$. Consistent with [13] and [15], we now delineate two inflammatory responses that play different roles in homeostasis or its loss.

\section{(i) Adaptive response}

To let inflammation engage during normal adaptations that promote tissue homeostasis and help to restore conditions when perturbed from normal [13,19], consider $\mu_{\varphi}(\tau)=k_{\varphi} \Upsilon_{\varphi}(\tau)$, where $\Upsilon_{\varphi}(\tau)=K_{\varphi}\langle\Delta \sigma(\tau)\rangle \geq 0$ is a stimulus function for inflammatory cell infiltration, with $K_{\varphi}$ an associated non-dimensional gain while the Macaulay brackets $\langle\cdot\rangle$ ensure that $\mu_{\varphi}(\tau) \geq 0 \forall \tau$ and, hence, $\Delta \varrho_{\varphi}(s) \geq 0 \forall s$ in Eq. (2.17). A value $\Delta \varrho_{\varphi}(s) \in[0,1]$, known from Eq. (2.17) at the current G\&R time $s$, enters the stimulus functions for smooth muscle and collagen production in Eq. (2.7), hence stimulating a combined (adaptive) immuno-mechano-driven mass turnover. Restoration 
of stresses to normal, along with the resolution of infiltration/activation rate $\mu_{\varphi}(\Delta \sigma)$, lead to an evolved mechano-adaptive homeostatic state.

\section{(ii) Maladaptive response}

Remarkably different characteristics manifest when inflammatory cells compromise homeostasis or drive the tissue toward disease. In particular, the onset of inflammation is typically delayed with respect to the mechano-adaptation [22], with the inflammatory response remaining "lockedin" for a certain period after the remodeling may be regarded complete [21]. In the present case of hypertension-induced aortic remodeling, the vessel can initially respond to an increase in pressure-induced wall stress by an (adaptive) mechano-driven mass turnover (as described in Section 2(b) i) that tries to restore the stress to normal and, only subsequently, an additional overriding inflammatory response arises (presumably) due to persistently high stresses, with inflammation remaining even if the stresses fall below normal during the (maladaptive) remodeling process; from a biological perspective, this secondary inflammatory response may relate to stress-mediated matrix damage or degradation, with persistent matrix fragments (e.g., exposed matricryptic sites) or altered matrix appearing as embedded neoantigens stimulating inflammatory activity [28]. Thus, for the rate of production (infiltration/activation), we assume that the inflammatory response promoting maladaptation is triggered only when the stress reaches a certain threshold [15]. Importantly, these combined features are not captured well with a mass production term that is proportional to a stress-dependent stimulus function (e.g., $\left.\Upsilon_{\varphi}=K_{\varphi}\langle\Delta \sigma\rangle\right)$, but demand a new approach for the rate of change of inflammation.

\section{(iii) Combined response}

Thus, let $\mu_{\varphi}(\tau) \geq 0$ be described by

$$
\begin{gathered}
\mu_{\varphi}(\tau)=k_{\varphi} K_{\varphi}\langle\Delta \sigma(\tau)\rangle \quad \text { if } \quad \sigma(\bar{\tau})<\sigma^{*} \quad \forall \bar{\tau} \leq \tau \\
\frac{d \mu_{\varphi}(\tau)}{d \tau}=\bar{\mu}_{\varphi}^{+} \quad \text { if } \quad \sigma(\tau) \geq \sigma^{*}, \\
\frac{d \mu_{\varphi}(\tau)}{d \tau}=-\bar{\mu}_{\varphi}^{-} \quad \text { if } \quad \sigma(\tau)<\sigma^{*} \quad \text { and } \quad \exists \bar{\tau}<\tau \mid \sigma(\bar{\tau}) \geq \sigma^{*},
\end{gathered}
$$

where $d \mu_{\varphi}(\tau) / d \tau=0$ otherwise and we delimit $\mu_{\varphi}(\tau) \in\left[0, k_{\varphi}\right] \forall \tau$. Here, $\bar{\mu}_{\varphi}^{+}>0$ and $\bar{\mu}_{\varphi}^{-}>0$ are constants and $\sigma^{*}$ is a (scalar metric of) stress threshold above which inflammatory cells infiltrate/activate during the maladaptive response; these parameters can be estimated from a measured time course for the inflammatory response and biaxial stresses, the latter relative to homeostatic set-points. The upper bound for $\mu_{\varphi}\left(\rightarrow k_{\varphi}\right)$ defines a saturation value which, if persistent, would eventually lead to a saturation value for the normalized-to-maximum density $\Delta \varrho_{\varphi}(\rightarrow 1)$ via Eq. (2.17); that is, with maximal infiltration $\mu_{\varphi}\left(=k_{\varphi}\right)$ being precisely offset by maximal removal $k_{\varphi} \Delta \varrho_{\varphi} \max \left(=k_{\varphi}\right)$ in Eq. (2.16). Importantly, inflammatory cell activity persists as long as $\mu_{\varphi}>0$, even if $\sigma$ drops below $\sigma^{*}$, though with decreasing intensity described by Eq. (2.20). Note that if $\sigma$ does not reach the inflammatory threshold $\sigma^{*}$, then $\mu_{\varphi}$ contributes to an adaptive immuno-mechano-mediated remodeling via Eq. (2.18), as in some cases of hypertension [29]. In general, a value $\Delta \varrho_{\varphi}(s) \in[0,1]$, known from Eq. (2.17) at the current G\&R time $s$, enters the stimulus functions for smooth muscle and collagen production in Eq. (2.7) and simultaneously modifies their inflammation-dependent passive properties in Eq. (2.9) [20,21], resulting in a coupled stress-driven immuno-mechanobiological response. Figure 2 (panel A) shows, schematically, how these coupled effects of stress and inflammation are integrated into the G\&R model. In addition, panel B shows that transitions among the mild adaptive (period I), acute maladaptive (II), saturated (III), and slow clearance (IV) inflammatory responses described by Eqs. (2.18)-(2.20) generally imply instantaneous changes in $d \mu_{\varphi}(\tau) / d \tau$, with both $\mu_{\varphi}(\tau)$ and $\Delta \varrho_{\varphi}(s)$ in Eq. (2.17) evolving continuously (the latter also smoothly) over time. Finally, by virtue of Eqs. (2.17)-(2.20), the current inflammatory cell density $\Delta \varrho_{\varphi}(s)$ depends on the past history 
of biaxial stresses $\sigma(\tau \leq s)$, or, in other words, there is not a one-to-one relationship between inflammation and stress, which will have important implications as noted below.

\section{(c) Parameter estimation}

In our previous studies [20,21], the time course of inflammatory cell density in Eq. (2.7) was prescribed based on experimental findings (CD45+ staining). We emphasize here that there remains a need for better time-course data, particularly at early times following the perturbation in loading. Nevertheless, a key outcome of many G\&R simulations of this type is correct prediction of long-term behaviors, which in mouse models of altered hemodynamics is typically after about two weeks. Here we use data available from a particular study [30,31] and model the inflammatory history constitutively in Eq. (2.17) along with (2.18)-(2.20), which requires additional determination of the parameters $\sigma^{*}, K_{\varphi}, k_{\varphi}, \bar{\mu}_{\varphi}^{+}$, and $\bar{\mu}_{\varphi}^{-}$. The remaining parameters in the model can be determined directly from experimental measurements (e.g., initial wall geometry, mass fractions, and in vivo state of stress and strain), nonlinear regressions from consistent biaxial mechanical data, and estimations based on immunomechanobiologically equilibrated evolutions over the course of AngII infusion; see Appendix in [20].

Here, $\sigma^{*}$ may be estimated as the value of $\sigma$ at the onset of the maladaptive inflammatory response. Let $\tau_{1}$ be the G\&R time at which $\sigma\left(\tau_{1}\right)=\sigma^{*}>\sigma_{o}$, with $\sigma$ generally increasing subsequently (see Figure 2, panel B, for definitions of time points). The stress $\sigma$ will remain greater than $\sigma^{*}$ over some period but will eventually equal $\sigma^{*}$ again (at $\tau_{3}$ ), and keep decreasing, consistent with the immuno-mechano-mediated turnover. Assume that the period for which $\sigma>\sigma^{*}$ persists is long enough such that the increasing inflammation rate saturates at $\mu_{\varphi}=k_{\varphi}$ (at $\tau_{2} \leq \tau_{3}$ ). Hence, neglecting an early, much milder increase in inflammation via Eq. (2.18) at time $\tau_{1}$, we find, from Eq. (2.19)

$$
\frac{d \mu_{\varphi}(\tau)}{d \tau}=\bar{\mu}_{\varphi}^{+} \Longrightarrow \mu_{\varphi}(\tau)=\bar{\mu}_{\varphi}^{+}\left(\tau-\tau_{1}\right) \quad \text { for } \quad \tau \in\left[\tau_{1}, \tau_{2}\right]
$$

with

$$
\frac{d \mu_{\varphi}(\tau)}{d \tau}=0 \Longrightarrow \mu_{\varphi}(\tau)=\bar{\mu}_{\varphi}^{+}\left(\tau_{2}-\tau_{1}\right) \equiv k_{\varphi} \quad \text { for } \quad \tau \in\left[\tau_{2}, \tau_{3}\right]
$$

and from Eq. (2.20)

$$
\frac{d \mu_{\varphi}(\tau)}{d \tau}=-\bar{\mu}_{\varphi}^{-} \Longrightarrow \mu_{\varphi}(\tau)=k_{\varphi}-\bar{\mu}_{\varphi}^{-}\left(\tau-\tau_{3}\right) \quad \text { for } \quad \tau>\tau_{3}
$$

Interestingly, consideration of this piecewise approximate solution for $\mu_{\varphi}(\tau)$ renders Eq. (2.17) integrable, yielding for $s \geq \tau$, where $s$ is the current G\&R time of interest,

$$
\begin{aligned}
\Delta \varrho_{\varphi}(s) & =\int_{\tau_{1}}^{s} \bar{\mu}_{\varphi}^{+}\left(\tau-\tau_{1}\right) e^{-k_{\varphi}(s-\tau)} d \tau \\
& =\frac{\bar{\mu}_{\varphi}^{+}}{k_{\varphi}}\left(s-\tau_{1}\right)-\frac{\bar{\mu}_{\varphi}^{+}}{k_{\varphi}^{2}}\left(1-e^{-k_{\varphi}\left(s-\tau_{1}\right)}\right) \text { for } s \in\left[\tau_{1}, \tau_{2}\right], \\
\Delta \varrho_{\varphi}(s) & =\int_{\tau_{1}}^{\tau_{2}} \bar{\mu}_{\varphi}^{+}\left(\tau-\tau_{1}\right) e^{-k_{\varphi}(s-\tau)} d \tau+\int_{\tau_{2}}^{s} k_{\varphi} e^{-k_{\varphi}(s-\tau)} d \tau \\
& =1-\frac{\bar{\mu}_{\varphi}^{+}}{k_{\varphi}^{2}}\left(e^{-k_{\varphi}\left(s-\tau_{2}\right)}-e^{-k_{\varphi}\left(s-\tau_{1}\right)}\right) \text { for } s \in\left[\tau_{2}, \tau_{3}\right],
\end{aligned}
$$


and

$$
\begin{aligned}
\Delta \varrho_{\varphi}(s)= & \int_{\tau_{1}}^{\tau_{2}} \bar{\mu}_{\varphi}^{+}\left(\tau-\tau_{1}\right) e^{-k_{\varphi}(s-\tau)} d \tau+\int_{\tau_{2}}^{\tau_{3}} k_{\varphi} e^{-k_{\varphi}(s-\tau)} d \tau \\
+ & \int_{\tau_{3}}^{s}\left(k_{\varphi}-\bar{\mu}_{\varphi}^{-}\left(\tau-\tau_{3}\right)\right) e^{-k_{\varphi}(s-\tau)} d \tau \\
= & 1-\frac{\bar{\mu}_{\varphi}^{+}}{k_{\varphi}^{2}}\left(e^{-k_{\varphi}\left(s-\tau_{2}\right)}-e^{-k_{\varphi}\left(s-\tau_{1}\right)}\right) \\
& -\frac{\bar{\mu}_{\varphi}^{-}}{k_{\varphi}}\left(s-\tau_{3}\right)+\frac{\bar{\mu}_{\varphi}^{-}}{k_{\varphi}^{2}}\left(1-e^{-k_{\varphi}\left(s-\tau_{3}\right)}\right) \text { for } s>\tau_{3}
\end{aligned}
$$

Note: if $\bar{\mu}_{\varphi}^{-}=0$, then $\Delta \varrho_{\varphi}$ approaches 1 (or $\bar{\mu}_{\varphi}^{+}\left(\tau_{3}-\tau_{1}\right) / k_{\varphi}$ if $\mu_{\varphi}<k_{\varphi}$ at $\tau_{3}$ ) for $s \gg \tau_{3}$, and remains locked-in regardless of the maladaptive stress drop. Conversely, if $\bar{\mu}_{\varphi}^{-}>0$, then $\Delta \varrho_{\varphi}$ reaches a maximum value $\lesssim 1$ (or $\lesssim \bar{\mu}_{\varphi}^{+}\left(\tau_{3}-\tau_{1}\right) / k_{\varphi}$ if $\mu_{\varphi}<k_{\varphi}$ at $\tau_{3}$ ) and subsequently decreases. In that case, Eqs. (2.24)-(2.26) allow the parameters $k_{\varphi}, \bar{\mu}_{\varphi}^{+}$, and $\bar{\mu}_{\varphi}^{-}$to be determined from a time course for the inflammatory response (e.g., given particular values for the early rate of change of $\Delta \varrho_{\varphi}(s)$, a potential peak $\Delta \varrho_{\varphi \max }$, and a long-term "remnant" value $\Delta \varrho_{\varphi}\left(s \gg \tau_{3}\right)$ ). Finally, the gain $K_{\varphi}$ can be estimated based on the modest increase in inflammation $\Delta \varrho_{\varphi}\left(s<\tau_{1}\right)$ prior to the onset of the maladaptive response.

\section{(d) Coupling methodology}

Equations (2.5) and (2.10) for the evolving mass density and stress of load-bearing constituents, along with Eqs. (2.3) and (2.7) for their mass production, Eqs. (2.4) and (2.6) for their removal, and Eqs. (2.8) and (2.9) for their passive response, are now coupled with Eqs. (2.17)-(2.20) for the evolving inflammatory activity through the stimulus function in Eq. (2.7) along with the possible presence of inflammation-dependent properties in Eq. (2.9) (see Supplemental Figure $\mathrm{S} 1$ for an extended schematic representation that includes all the model equations and their couplings). Hence, at each G\&R time step, the following algorithmic implementation can ensure simultaneous satisfaction of these equations before advancing to the next G\&R time step:

I. With constituent stresses given by Eqs. (2.10) and mass (density) fractions from Eqs. (2.5), enforce mechanical equilibrium under current internal pressure and axial stretch, with fixed medial and adventitial Jacobians $J_{\Gamma}$, to compute the vessel geometry and biaxial stresses at the current time $s$.

II. Compute and store the inflammatory cell infiltration $\mu_{\varphi}(\tau=s)$ via Eqs. (2.18)-(2.20), whose past history $\mu_{\varphi}(\tau<s)$ is needed to subsequently compute the current value for $\Delta \varrho_{\varphi}(s)$ via Eq. (2.17).

III. Update inflammation-dependent elastic parameters for collagen fibers and smooth muscle cells in Eq. (2.9).

IV. Update rate parameters (2.6) and stimulus functions (2.7) to compute mass removal (2.4) and production (2.3) using mass densities from the previous iteration.

V. Compute mass densities via Eq. (2.5) and update associated medial and adventitial Jacobians $J_{\Gamma}=\sum \rho_{\Gamma \mathrm{R}}^{\alpha} / \rho$.

VI. Repeat from step (I) with updated variables until the error between iterative mass densities is lower than a prescribed tolerance.

This iterative procedure converges to the exact (implicit) solution at the current G\&R time $s$ after few iterations. The integration scheme becomes explicit if one stops at step $(\mathrm{V})$ and proceeds to the next time step without iterating. 


\section{Results}

According to the present modeling approach, a marked (chronic) increase in $\Delta \varrho_{\varphi}$ via Eq. (2.16) requires an early increase in the cell infiltration rate $\mu_{\varphi}$ via Eq. (2.19) which in turn requires the stress $\sigma$ to remain above the threshold $\sigma^{*}$ for a certain period (of the order of days to weeks). Hence, the higher and more persistent the stress at the tissue level, the greater the inflammation. Therefore, effects that reduce the magnitude and/or duration of a peak in the in-plane biaxial stresses (as, for example, experienced early in hypertension-induced protocols) could reduce the extent of inflammation and whether it promotes or prevents mechanical homeostasis at the tissue level.

\section{(a) Increase in blood pressure}

In this example we verify and validate the present coupled formulation by reproducing previous experimental [31] and computational [20] results on early hypertension-induced remodeling of the aorta of male wild-type (C57BL/6) mice that focused on changes in the passive mechanical behavior induced by changes in extracellular matrix. Consideration of different temporal profiles for pressure elevation allows us to predict additional results enabled by the novel coupling between stress and inflammation proposed herein. Geometrical, mechanical, and G\&R parameters are taken from [20] without modification, see Table 1. Consistent with the 4-week (discrete) time course of the study and the biaxial stresses reported in [31], we estimate $\sigma^{*} \equiv \sigma_{v}^{*}=$ $170 \mathrm{kPa}$ (with $\sigma \equiv \sigma_{v}=(\operatorname{tr} \boldsymbol{\sigma}) / 3=(\boldsymbol{\sigma}: \mathbf{I}) / 3$ the volumetric stress), $K_{\varphi}=2.5, k_{\varphi}=2 / 7$ days $^{-1}$, and $\bar{\mu}_{\varphi}^{+}=0.102$ days $^{-2}$, with the (long-term) parameter $\bar{\mu}_{\varphi}^{-}=0$, see Section 2(c). Eqs. (2.17)-(2.20) for the evolving inflammation can then be integrated numerically while advancing the coupled simulations, here with a time step $\Delta s=\left(1 / k_{\varphi}\right) / 10=0.35$ days and a relative error tolerance of $10^{-9}$, see Section $2(\mathrm{~d})$.

Figure 3 shows predictions of the present coupled model for a prescribed 1.36-fold increase in systolic pressure achieved over a period of 7 days and maintained to 28 days (panel A, solid lines), as in [20] based on experimental measurements. This rapid increase in pressure provokes a relatively high early increase in circumferential (I) and axial (J) stresses that induce an, herein computed, inflammatory response that eventually reaches the maximum value $\Delta \varrho_{\varphi \max }=1(\mathrm{~L})$, consistent with the prior experimental observations. To illustrate the coupling between stress and inflammation within the present approach, panel K shows how evolution of the mean volumetric stress $\sigma \equiv \sigma_{v}>\sigma_{v}^{*}$ stimulates a rapid increase in the inflammatory term $\mu_{\varphi}$ via Eq. (2.19) that subsequently triggers $\Delta \varrho_{\varphi}$ via Eq. (2.17), adversely affecting the remodeling. Indeed, note the highly maladaptive response, with intramural stresses much lower than normal after two weeks of AngII infusion, consistent with an excessive adventitial thickening ( $\mathrm{H}$, compare to medial thickening, D) caused by fibrosis in the adventitia (F, G, compare to medial smooth muscle hyperplasia/hypertrophy, B, C; [31]), with a mild decrease in luminal radius (E). Noting that slower increases in pressure up to the same hypertensive systolic pressure would decrease the peak for intramural stresses (because the tissue can effectively respond / remodel faster relative to the stimulation timescale, cf. [24]), we progressively extended in silico the period over which the pressure increases from 7 to 21 days. Predictions for these increasing periods $(7,14,18$, and 21 days to reach the 1.36-fold increase in pressure) show how slower hypertensive progression results in a reduced maximal intramural stress $\sigma_{v}$ and associated reduced maximal induced inflammation $\Delta \varrho_{\varphi}(s=28$ days $)$, thus allowing progressively better mechano-adaptations with reduced adventitial fibrosis and hence thickening. In particular, for the slowest simulated increase in pressure, achieved over 21 days (dotted lines), intramural stresses do not reach the inflammatory threshold stress, and the model predicts an adaptive, both immuno- and mechano-mediated, remodeling response to the same level of hypertension. Consistent with Eq. (2.18), a modest inflammatory response, stimulated by a modest increase in biaxial stresses, arises during the adaptation, hence supporting a mechanical homeostasis through an additional negative feedback mechanism, via Eq. (2.7), that helps return both perturbations within normal 
homeostatic ranges $(\mathrm{K}, \mathrm{L}$, dotted lines; note that inner radius, hence shear stress stimulus for constant cardiac output, also returns to normal, E). Indeed, albeit not shown, deactivation of this initially supportive immuno-driven turnover (i.e., with $K_{\varphi}=0$ in Eq. (2.18)) for the simulation with the slowest increase in pressure predicts a slower remodeling (relative to $K_{\varphi}=2.5$ ) with $\Delta \varrho_{\varphi}=0$ initially, whereby stresses slightly higher than the threshold for a short period result in a modest maladaptive response.

\section{(b) Increase in contractility}

Experimental findings demonstrate an important role of smooth muscle mediated vasomotor control of vessel lumen in flow-induced remodeling [7,16], and it appears that contractility plays a similarly important role in hypertensive remodeling [22,32]. Nevertheless, the precise role of contractility in hypertensive aortic remodeling remains unclear due in part to lack of information on the basal tone in vivo and to what degree tone changes in hypertension. In this example we explore, in silico, the effects of different levels of smooth muscle contractility added to the passive response considered in the previous example. For illustrative purposes, we let $T_{\max }=258 \mathrm{kPa}$, $\lambda_{\mathrm{M}}=1.1, \lambda_{0}=0.6, C_{\mathrm{B}}=0.833, C_{\mathrm{S}}=1.666$, and $k_{\text {act }}=1 / 7$ days $^{-1}$ in Eqs. (2.12) and (2.13) (Table 1 , cf. $[24,33])$. All remaining parameters remain the same as in Example 3(a).

Importantly, given an in vivo systolic pressure, inclusion of this additional contribution to the total stress $\sigma$ modifies the homeostatic state from which subsequent G\&R simulations should be initiated (consistent with initially equilibrated stimulus functions $\Upsilon_{\Gamma}^{\alpha}=1$ in Eq. (2.7)). In particular, basal smooth muscle contraction reduces inner radius and increases medial and adventitial thicknesses, with circumferential and axial stress decreasing from $\sigma_{\theta \theta o}^{(\text {pas })}=231 \mathrm{kPa}$ and $\sigma_{z z o}^{(p a s)}=258 \mathrm{kPa}$ (see G\&R time $s=0$ in Figure 3) to $\sigma_{\theta \theta o}=205 \mathrm{kPa}$ and $\sigma_{z z o}=207 \mathrm{kPa}$. Despite a lack of additional experimental data for the subsequent remodeling under constant pressure, this contracted state is assumed herein for illustrative purposes to correspond to a homeostatic state that enables subsequent G\&R simulations to include both passive and active contributions to stress from G\&R time $s=0$. Starting from this state, we let the pressure increase over 7 days to the level that caused maximal inflammation in the previous example (solid lines in Figure 3), which in this case resulted in a peak (passive plus active) stress $\sigma_{v \max }=$ $155 \mathrm{kPa}<170 \mathrm{kPa}=\sigma_{v}^{*}$ at day 5 , and, therefore, did not trigger a chronic inflammatory response and associated maladaptation; that is, the remodeling led to an adaptive immuno-mechanoadaptation.

Although a protective role of contractility was expected since it reduces the intramural stress state [22,32], consider now a (fictitious) reference simulation for which an increase in pressure over 7 days causes maximal inflammation when both passive and (baseline) active contributions to stress are considered from the onset of hypertension, for which we consider a reduced inflammatory stress threshold $\sigma_{v}^{*}=150 \mathrm{kPa}<170 \mathrm{kPa}$. Since $\sigma_{v \max }=155 \mathrm{kPa}>150 \mathrm{kPa}=\sigma_{v}^{*}$, this numerical experiment allows us to explore in silico the potentially protective role of an active tone. Moreover, to consider the possibility that contractile strength could increase in hypertension (e.g., smooth muscle hypertrophy), consider an increase up to AngII hypertension-appropriate levels to overcome potentially emerging, adverse, inflammatory effects (cf. [34]). To facilitate comparisons relative to a common baseline contracted state, let $T_{\max }$ in Eq. (2.12) increase with the extent of hypertension as $T_{\max }(s)=T_{\max }(0)\left(1+K_{P}(P(s)-P(0)) / P(0)\right)$, with $K_{P}$ a gain parameter, with $T_{\max }(0)=258 \mathrm{kPa}$ as in the prior example. Figure 4 shows predictions for a 1.36fold increase in systolic pressure over 7 days (A) for $K_{P}=0$ (i.e., $T_{\max }(s)=T_{\max }(0)=258 \mathrm{kPa}$ remains constant; solid lines), for which an early increase in stress $\sigma_{v}>\sigma_{v}^{*}(\mathrm{~K})$ induces an inflammatory response that approaches the maximum value $\Delta \varrho_{\varphi \max }=1(\mathrm{~L})$. Similar to the case where only passive stresses were considered (cf. solid lines in Figure 3), a highly maladaptive response (with low intramural stresses, I, J, consistent with an excessive adventitial thickening, $\mathrm{H}$, mainly caused by an aberrant deposition of collagen in the adventitia, F, G) emerges, which highlights that chronic inflammation, once present, can overcome the potentially protective 
role of contractility and strengthens the idea of its overriding role in disease progression [15]. Simulations with higher values for the hypertensive gain parameter $K_{P}$ predict gradual reductions in the maximal intramural stress $\sigma_{v}$ and associated maximal induced inflammation $\Delta \varrho \varphi(s=28$ days), suggesting a potentially (pre-emptively) protective role of heightened smooth muscle contractility, with enhanced contractile responses preventing the vessel from experiencing high stresses that can trigger an adverse inflammatory response. In particular, for the highest regulation of tone considered, $K_{P}=4$ (dotted lines), the mean intramural stress $\sigma_{v}$ did not reach the inflammatory threshold $\sigma_{v}^{*}$ and the model predicted an adaptive, immuno-mechanomediated remodeling response, with an early increase in inflammation reversed during the subsequent adaptation to the same level of hypertension. Importantly, note that the prescribed increase in pressure combined with increasing contractile properties resulted in increasing peaks of circumferential stress $\sigma_{\theta \theta}$ during an early remodeling stage, hence suggesting that $\sigma_{\theta \theta}$ alone would not be a good metric for the present G\&R model with stress-driven inflammation and highlighting, in turn, the importance of considering a metric (e.g., $\sigma_{v}$ ) that assesses the biaxial nature of the tensional state in the arterial wall. Nevertheless, there is a need for more experimental information on the potential role of axial stress in arterial remodeling.

\section{(c) Persistent long-term inflammatory response}

Our immuno-mechanical aortic modeling [21] of atheroprone mice subjected to AngII-induced hypertension for 28 days followed by 7 months of recovery without AngII infusion [22] predicted that inflammation persisted, in part, for long periods after removing the exogenous AngII stimulus. A subsequent re-evaluation of experimental data confirmed this prediction. This partial reversal of the (maladaptive) inflammatory response can be described within the present coupled model by considering the long-term parameter $\bar{\mu}_{\varphi}^{-}>0$ in Eq. (2.20). In particular, similar to the computational prediction, and experimental verification, assume that a remnant inflammatory cell density $\Delta \varrho_{\varphi} \approx 0.4$ remains at day 224 following the 28 days of induced hypertension. Indeed, with all requisite values known from the reference simulation in Example 3(a) $\left(\tau_{1} \approx 2\right.$ days, $\tau_{2} \approx \tau_{3} \approx 5$ days, $k_{\varphi}=2 / 7$ days $^{-1}$, and $\bar{\mu}_{\varphi}^{+}=0.102$ days $\left.^{-2}\right)$, Eq. (2.26), with $\Delta \varrho \varphi(s=224$ days $) \approx$ 0.4 suggests a value $\bar{\mu}_{\varphi}^{-} \approx 0.0008$ days $^{-2}$, which we then prescribed as an input parameter to our coupled model. Also motivated by experimental observations in [22], we prescribed a slow decrease in systolic pressure to $P / P_{o}=1.15$ (down from a maximal 1.36 in [20], similar to the decrease to 1.26 from a maximal 1.68 in [21]).

Figure 5 (solid lines) extends the results predicted in Figure 3 (solid lines) for wild-type (C57BL/6) mice up to 224 days, with the model predicting a linear decrease in inflammation from its maximal value at day 28 to the "experimental" value 0.4 at day 224 (L). Due to the lower inflammation and reduced pressure (A), medial (D), but especially adventitial (H), thickening (hence stresses, I, J) are substantially restored toward hypertensive-appropriate values, although the vessel still remains overly-thick relative to the remnant pressure $P / P_{O}=1.15$ due to the persistent inflammation. This partially reversible slow adaptation is possible numerically because we let the inflammation-dependent parameters for smooth muscle and collagen fibers return toward normal values in line with the decrease in inflammation. Yet, experimental observations in [22], as well as associated computations in [21], suggest that even if inflammation has partially resolved and pressure dropped, the long-term response yet remains markedly maladaptive, with both thickness and stresses remaining far from the initial homeostatic values. Hence, we also show results (dashed lines) for a simulation where the computed inflammation decreases during the long period without AngII infusion (i.e., we maintain the value $\bar{\mu}_{\varphi}^{-}>0$ ), which affects the stimulus functions in Eq. (2.7), but with the smooth muscle and collagen fiber parameters retained as fully maladaptive (i.e., those associated with $\Delta \varrho_{\varphi \max }$ ), which adversely affect their passive properties and yields a more realistic long-term maladaptation consistent with [22] and [21]. 


\section{Discussion}

There are many different models of hypertension in mice, but infusion of AngII has emerged as one of the most common given its diverse but highly reproducible effects on central arteries. Importantly, AngII increases total peripheral resistance and thereby elevates central blood pressure, thus increasing the hemodynamic load on the central arteries; in addition, it stimulates significant vascular inflammation throughout the vasculature. Macrophages, both resident and recruited, play key roles in AngII-induced hypertensive aortic remodeling as evidenced by measurements of cellular infiltration within the aortic wall and especially by experiments wherein reductions of recruited macrophages or their activity, including via CCR2 receptor disruption, attenuates otherwise marked aortic remodeling [35-37]. Notwithstanding the tremendous insight gained via such in vivo experiments, the potentially overlapping effects of increased mechanical loading and stimulation of inflammation makes it difficult to delineate mechanisms. We know, for example, that increased mechanical loading (stretching/stressing) of isolated smooth muscle cells and fibroblasts changes gene expression to promote heightened matrix turnover [30,38], with mechanical stretch similarly able to induce pro-inflammatory genes in isolated macrophages [39,40]. Conversely, exposure of isolated medial smooth muscle cells to exogenous AngII stimulates cell production of interleukin-6 (IL-6) whereas exposure of adventitial fibroblasts to AngII stimulates production of both monocyte chemoattractant protein-1 (MCP-1), which attracts monocytes, and IL-6, which activates monocytes [41,42]. Exogenous AngII also directly stimulates matrix production by isolated smooth muscle cells and fibroblasts $[43,44]$, and mechanical loading stimulates local production of AngII by smooth muscle cells [38,45], which contributes to matrix production (synthesis) and removal (degradation by MMPs). Importantly, disruption of the $\alpha_{1}$ integrin subunit disrupts mechano-sensing and attenuates AngII-induced elastic artery remodeling [46]. Pressure-induced mechanical stress and exogenous AngII can thus have both separate and synergistic effects on aortic remodeling [47,48], highlighting the complex interactions amongst mechanical stress and inflammation in driving matrix turnover.

T-cells similarly play multiple roles in AngII-induced hypertension and associated aortic remodeling. Among other effects, T-cells accumulate in perivascular fat and to a lesser extent in the aortic wall following AngII infusion. They increase oxidative stress and contribute to adventitial fibrosis by stimulating collagen production [49], the latter due in part to production of interleukin-17a (IL-17a), which has been shown in cell culture to directly stimulate collagen production by isolated adventitial fibroblasts, which of course also increase collagen production in response to increased stretch [30]. These T-cells appear to produce interferon gamma (IFN- $\gamma$ ) and tissue necrosis factor alpha (TNF- $\alpha$ ) at a normal rate in AngII-induced hypertension, though the intramural amounts of these two pro-inflammatory cytokines is greater in hypertension due to the significant increase in the number of recruited cell [50]. Importantly, it was shown further that effects of AngII-induced hypertension on T-cells in (humanized) mice was due to the "hypertensive mileu" and not the direct action of AngII on the T-cells [51], consistent with the aforementioned concept that inflammation arises in support of homeostasis when primary homeostatic processes are insufficient to reduce the effects of the perturbation quickly enough [13]. Notwithstanding the extreme complexities of inflammation in hypertension [52-54], which are not yet understood fully even for AngII-induced hypertension, it is clear that one must consider both immuno- and mechano-contributions to the associated aortic remodeling.

This paper was motivated primarily by particular experimental and computational findings of AngII-induced remodeling of the descending thoracic aorta in male C57BL/ 6 and Apoe ${ }^{-/}$mice on a C57BL/ 6 background. We had observed a trend toward mechano-adaption of this segment of the aorta up to 14 days of high rate AngII infusion, but a remarkable maladaptive response thereafter that was characterized by a dramatic infiltration of CD45+ cells, including CD3+ T-cells, and CD68+ macrophages that correlated with a marked accumulation of collagen, especially in the adventitia. These findings suggested that a mechano-driven remodeling preceded immunomediated remodeling [22]. That a normal (mechanical) homeostatic process responds first to a 
perturbation in pressure and is then followed by an inflammatory process is consistent with the concept put forth by [13] that inflammation can support homeostasis when the primary restorative mechanisms are insufficient. Yet, in this case, the inflammation drove a maladaptive, not adaptive, remodeling of the aorta. We later attempted to model this complex aortic response and found for the first time that gain parameters and set-points within a mechanobiological model needed to evolve to fit this complex data set [21]. This change in homeostatic parameters is also consistent with the observation of [15] that, being a prioritized process, inflammation can override normal homeostatic set-points and gains. Whereas we previously prescribed the time-course of the inflammation that drove the associated remodeling, here we have introduced a new approach consistent with the concept of [15] whereby mechanical stress induces chronic inflammation only if particular thresholds are reached. Remarkably, if stresses do not reach these thresholds, this approach also allows lower levels of inflammation to emerge to support mechanical homeostasis; it is therefore consistent, too, with the concept of para-inflammation put forth by [13].

The Laplace equation illustrates well the fundamental role of distending pressure on the value of mean circumferential wall stress, but it is seldom emphasized that smooth muscle cell tone plays a similarly fundamental role. That is, if we write this equation as $\sigma_{\theta \theta}=P a(P, C) / h(P, C)$, where $a$ and $h$ are luminal radius and wall thickness, respectively, and $P$ and $C$ are the distending pressure and contractile strength, then it is clear that pressure and smooth muscle tone are equally important determinants of intramural stress, which we include as a convenient homeostatic target (actually the first invariant of stress). Accumulating evidence suggests strongly that increased local smooth muscle contractility dramatically reduces the degree of hypertensive remodeling $[22,32,34]$. We thus included the role of smooth muscle tone as a physiological modifier of the mechanical stress stimulus, and indeed found that increasing smooth muscle tone reduces the degree of the remodeling response by attenuating the increase in intramural stress due to pressure elevation. We also found that a sufficiently high level of smooth muscle tone can prevent the engagement of inflammation despite infusion of the pro-inflammatory peptide AngII, consistent with differential findings between the non-contractile thoracic and highly contractile abdominal aorta [22] as well as the finding of reduced inflammation in AngII-induced hypertension in the presence of blood pressure (and thus intramural stress) lowering drugs [51]. Among other key predictions, the computationally modeled aorta was better able to respond to the same foldincrease in pressure for lower rates of pressure elevation. That is, it is easier to adapt to a slower, progressive increase in pressure than to an abrupt increase, with stress-induced inflammation playing a key role in the underlying remodeling. This finding is similar to that predicted by purely mechanobiological models for vein graft remodeling under extreme changes in hemodynamic conditions [55] and is intuitive given that changes in gene expression and translation into functional structural modifications of the wall are time-dependent and the cells have finite limits on their rates of division or production. Hence, it also appropriately extends our previous analysis of critical roles that different timescales may have on ideal mechano-adaptations [24] by taking into account alternate, either reversible or overriding, effects of inflammation within a generally coupled remodeling scenario. It was also found that an initial (para)inflammatory response contributes to the mechano-adaptation, accelerating the remodeling and helping to reduce maximal wall stresses for the same perturbation in pressure, hence highlighting a key contribution of this additional mechanism in promoting homeostasis, especially when purely mechano-driven turnover mechanisms are unable to respond quickly enough to recover from a (otherwise mild) perturbation. Finally, it was found that, even if both pressure and inflammation are partially reversed after several months of recovery without AngII infusion, the mechanical properties of the newly deposited constituents during this long period need not return to normal values at the same pace, hence suggesting that the ensuing remodeling depends not only on the current, persistent level of inflammation but, more generally, on the longer, past history of inflammation. It is for this reason that convolution-integral based relations (e.g., 2.17) proved useful. 
In conclusion, it has long been known that the aorta adapts in response to modest sustained changes in hemodynamic loading via cell and matrix turnover within evolving configurations. It is becoming increasingly evident that inflammation plays either complementary or contrasting roles with such mechano-adaptations, with modest inflammation promoting homeostatic remodeling, but marked or chronic inflammation preventing homeostasis and driving disease progression. We have introduced the first coupled immuno-mechano model of aortic remodeling, consistent with new concepts of inflammation as well as data from a common mouse model of hypertension. As with many other phenomenological models of vascular growth and remodeling, the present model is descriptive but also predictive; it can be used to generate and test new hypotheses and to guide experimental design. Nevertheless, there is also a need for more mechanistic modeling, as, for example, incorporating appropriate cell signaling models (cf. [56]) within the current continuum framework to enable modeling from transcript to tissue.

Data Accessibility. The code that replicates all study findings reported in the article is available at https:/ / github.com/cmm-gr/stress-driven-inflammation.git

Authors' Contributions. ML: Conceptualization, Methodology, Software, Formal analysis, Investigation, Data curation, Writing/editing original draft, Visualization. BS: Conceptualization, Methodology, Investigation, Writing/editing original draft, Visualization. JH: Conceptualization, Methodology, Investigation, Writing/editing original draft, Visualization, Supervision, Project administration, Funding acquisition. All authors gave final approval for publication and agree to be held accountable for the work performed therein.

Competing Interests. The authors declare no conflict of interest, financial or otherwise.

Funding. This work was supported, in part, by grants from the US National Institutes of Health (R01 HL105297, P01 HL134605, R01 HL146723).

Acknowledgements. We thank Professor Matthew R. Bersi, Washington University in St. Louis, for thoughtful discussions on aortic remodeling in hypertension.

\section{References}

1. Humphrey JD. 2001 Stress, strain, and mechanotransduction in cells. J. Biomech. Eng. 123, 638641.

2. Balaban NQ, Schwarz US, Riveline D, Goichberg P, Tzur G, Sabanay I, Mahalu D, Safran S, Bershadsky A, Addadi L, Geiger B. 2001 Force and focal adhesion assembly: a close relationship studied using elastic micropatterned substrates. Nature cell biology 3, 466-472.

3. Tan JL, Tien J, Pirone DM, Gray DS, Bhadriraju K, Chen CS. 2003 Cells lying on a bed of microneedles: an approach to isolate mechanical force. Proceedings of the National Academy of Sciences 100, 1484-1489.

4. Rudic RD, Shesely EG, Maeda N, Smithies O, Segal SS, Sessa WC. 1998 Direct evidence for the importance of endothelium-derived nitric oxide in vascular remodeling. The Journal of clinical investigation 101, 731-736.

5. Baeyens N, Bandyopadhyay C, Coon BG, Yun S, Schwartz MA. 2016 Endothelial fluid shear stress sensing in vascular health and disease. The Journal of clinical investigation 126, 821-828.

6. Clark JM, Glagov S. 1985 Transmural organization of the arterial media. The lamellar unit revisited.. Arteriosclerosis 5, 19-34.

7. Dajnowiec D, Langille BL. 2007 Arterial adaptations to chronic changes in haemodynamic function: coupling vasomotor tone to structural remodelling. Clinical science 113, 15-23.

8. Humphrey JD. 2008 Vascular adaptation and mechanical homeostasis at tissue, cellular, and sub-cellular levels. Cell Biochemistry and Biophysics 50, 53-78.

9. Wynn TA, Chawla A, Pollard JW. 2013 Macrophage biology in development, homeostasis and disease. Nature 496, 445-455.

10. Okabe Y, Medzhitov R. 2016 Tissue biology perspective on macrophages. Nature immunology $17,9$.

11. Joshi H, Morley SC. 2019 Cells under stress: the mechanical environment shapes inflammasome responses to danger signals. Journal of Leukocyte Biology 106, 119-125.

12. Jain N, Moeller J, Vogel V. 2019 Mechanobiology of macrophages: how physical factors coregulate macrophage plasticity and phagocytosis. Annual review of biomedical engineering 21, 
267-297.

13. Chovatiya R, Medzhitov R. 2014 Stress, inflammation, and defense of homeostasis. Molecular cell 54, 281-288.

14. Medzhitov R. 2008 Origin and physiological roles of inflammation. Nature 454, 428-435.

15. Kotas ME, Medzhitov R. 2015 Homeostasis, inflammation, and disease susceptibility. Cell 160, 816-827.

16. Bakker EN, Matlung HL, Bonta P, De Vries CJ, Van Rooijen N, VanBavel E. 2008 Blood flowdependent arterial remodelling is facilitated by inflammation but directed by vascular tone. Cardiovascular research 78, 341-348.

17. Tang PC, Qin L, Zielonka J, Zhou J, Matte-Martone C, Bergaya S, van Rooijen N, Shlomchik WD, Min W, Sessa WC, Pober JS, Tellides G. 2008 MyD88-dependent, superoxide-initiated inflammation is necessary for flow-mediated inward remodeling of conduit arteries. The Journal of experimental medicine 205, 3159-3171.

18. Zhou J, Tang PC, Qin L, Gayed PM, Li W, Skokos EA, Kyriakides TR, Pober JS, Tellides G. 2010 CXCR3-dependent accumulation and activation of perivascular macrophages is necessary for homeostatic arterial remodeling to hemodynamic stresses. Journal of Experimental Medicine 207, 1951-1966.

19. Tellides G, Pober JS. 2015 Inflammatory and immune responses in the arterial media. Circulation Research 116, 312-322.

20. Latorre M, Humphrey JD. 2018 Modeling mechano-driven and immuno-mediated aortic maladaptation in hypertension. Biomechanics and Modeling in Mechanobiology 17, 1497-1511.

21. Latorre M, Bersi MR, Humphrey JD. 2019 Computational modeling predicts immunomechanical mechanisms of maladaptive aortic remodeling in hypertension. International Journal of Engineering Science 141, 35-46.

22. Bersi MR, Khosravi R, Wujciak AJ, Harrison DG, Humphrey JD. 2017 Differential cellmatrix mechanoadaptations and inflammation drive regional propensities to aortic fibrosis, aneurysm or dissection in hypertension. Journal of The Royal Society Interface 14, 20170327.

23. Baek S, Valentín A, Humphrey JD. 2007 Biochemomechanics of cerebral vasospasm and its resolution: II. Constitutive relations and model simulations. Annals of Biomedical Engineering 35, 1498-1509.

24. Latorre M, Humphrey JD. 2018 Critical roles of time-scales in soft tissue growth and remodeling. APL Bioengineering 2, 026108.

25. Cyron CJ, Humphrey JD. 2014 Vascular homeostasis and the concept of mechanobiological stability. International journal of engineering science 85, 203-223.

26. Latorre M, Humphrey JD. 2019 Mechanobiological stability of biological soft tissues. Journal of the Mechanics and Physics of Solids 125, 298-325.

27. Hill MR, Philp CJ, Billington CK, Tatler AL, Johnson SR, O'Dea RD, Brook BS. 2018 A theoretical model of inflammation- and mechanotransduction-driven asthmatic airway remodelling. Biomechanics and modeling in mechanobiology 17, 1451-1470.

28. Davis GE, Bayless KJ, Davis MJ, Meininger GA. 2000 Regulation of tissue injury responses by the exposure of matricryptic sites within extracellular matrix molecules. The American journal of pathology 156, 1489-1498.

29. Humphrey JD. 2008 Mechanisms of arterial remodeling in hypertension. Hypertension 52, 195 200.

30. Wu J, Thabet SR, Kirabo A, Trott DW, Saleh MA, Xiao L, Madhur MS, Chen W, Harrison DG. 2014 Inflammation and Mechanical Stretch Promote Aortic Stiffening in Hypertension Through Activation of p38 Mitogen-Activated Protein Kinase. Circulation Research 114, 616625.

31. Bersi MR, Bellini C, Wu J, Montaniel KRC, Harrison DG, Humphrey JD. 2016 Excessive adventitial remodeling leads to early aortic maladaptation in angiotensin-induced hypertension. Hypertension 67, 890-896.

32. Korneva A, Humphrey JD. 2019 Maladaptive aortic remodeling in hypertension associates with dysfunctional smooth muscle contractility. American Journal of Physiology 316, H265$\mathrm{H} 278$.

33. Ahmadzadeh H, Rausch MK, Humphrey JD. 2018 Particle-based computational modelling of arterial disease. Journal of the Royal Society Interface 15, 20180616.

34. Spronck B, Latorre M, Mehta S, Caulk AW, Ramachandra AB, Murtada SI, Rojas A, He CS, Jiang B, Wang M, Bersi MR, Tellides G, Humphrey JD. 2020 Genetic Background Dictates Aortic Fibrosis in Hypertensive Mice. Preprint at https://doi.org/10.1101/727800. 
35. Bush E, Maeda N, Kuziel WA, Dawson TC, Wilcox JN, DeLeon H, Taylor WR. 2000 CC chemokine receptor 2 is required for macrophage infiltration and vascular hypertrophy in angiotensin II-induced hypertension. Hypertension 36, 360-363.

36. Ishibashi M, Hiasa Ki, Zhao Q, Inoue S, Ohtani K, Kitamoto S, Tsuchihashi M, Sugaya T, Charo IF, Kura S et al.. 2004 Critical role of monocyte chemoattractant protein-1 receptor CCR2 on monocytes in hypertension-induced vascular inflammation and remodeling. Circulation research $\mathbf{9 4}, 1203-1210$.

37. Moore JP, Vinh A, Tuck KL, Sakkal S, Krishnan SM, Chan CT, Lieu M, Samuel CS, Diep H, Kemp-Harper BK, Tare M, Ricardo SD, Guzik TJ, Sobey CG, Drummond GR. 2015 M2 macrophage accumulation in the aortic wall during angiotensin II infusion in mice is associated with fibrosis, elastin loss, and elevated blood pressure. American Journal of Physiology-Heart and Circulatory Physiology 309, H906-H917.

38. Li Q, Muragaki Y, Hatamura I, Ueno H, Ooshima A. 1998 Stretch-induced collagen synthesis in cultured smooth muscle cells from rabbit aortic media and a possible involvement of angiotensin II and transforming growth factor- $\beta$. Journal of vascular research 35, 93-103.

39. Adams S, Wuescher LM, Worth R, Yildirim-Ayan E. 2019 Mechano-Immunomodulation: Mechanoresponsive Changes in Macrophage Activity and Polarization. Annals of Biomedical Engineering 47, 2213-2231.

40. Meli VS, Veerasubramanian PK, Atcha H, Reitz Z, Downing TL, Liu WF. 2019 Biophysical regulation of macrophages in health and disease. Journal of leukocyte biology 106, 283-299.

41. Han Y, Runge MS, Brasier AR. 1999 Angiotensin II induces interleukin-6 transcription in vascular smooth muscle cells through pleiotropic activation of nuclear factor- $\kappa \mathrm{B}$ transcription factors. Circulation research 84, 695-703.

42. Tieu BC, Ju X, Lee C, Sun H, Lejeune W, Recinos III A, Brasier AR, Tilton RG. 2011 Aortic adventitial fibroblasts participate in angiotensin-induced vascular wall inflammation and remodeling. Journal of vascular research $48,261-272$.

43. Ford CM, Li S, Pickering JG. 1999 Angiotensin II stimulates collagen synthesis in human vascular smooth muscle cells: involvement of the AT1 receptor, transforming growth factor- $\beta$, and tyrosine phosphorylation. Arteriosclerosis, thrombosis, and vascular biology 19, 1843-1851.

44. Che Zq, Gao Pj, Shen Wl, Fan Cl, Liu JJ, Zhu Dl. 2008 Angiotensin II-stimulated collagen synthesis in aortic adventitial fibroblasts is mediated by connective tissue growth factor. Hypertension Research 31, 1233-1240.

45. Williams B, O'Callaghan CJ. 2000 Mechanical strain-induced extracellular matrix production by human vascular smooth muscle cells: role of TGF- $\beta 1$. Hypertension 36, 319-324.

46. Louis H, Kakou A, Regnault V, Labat C, Bressenot A, Gao-Li J, Gardner H, Thornton SN, Challande P, Li Z, Lacolley P. 2007 Role of $\alpha 1 \beta 1$-integrin in arterial stiffness and angiotensininduced arterial wall hypertrophy in mice. American Journal of Physiology-Heart and Circulatory Physiology 293, H2597-H2604.

47. Bardy N, Merval R, Benessiano J, Samuel JL, Tedgui A. 1996 Pressure and angiotensin II synergistically induce aortic fibronectin expression in organ culture model of rabbit aorta: evidence for a pressure-induced tissue renin-angiotensin system. Circulation Research 79, 70-78.

48. Spronck B, Ferruzzi J, Bellini C, Caulk AW, Murtada SI, Humphrey JD. 2020 Aortic remodeling is modest and sex-independent in mice when hypertension is superimposed on aging. Journal of Hypertension 38, 1312-1321.

49. Guzik TJ, Hoch NE, Brown KA, McCann LA, Rahman A, Dikalov S, Goronzy J, Weyand C, Harrison DG. 2007 Role of the T cell in the genesis of angiotensin II-induced hypertension and vascular dysfunction. Journal of experimental medicine 204, 2449-2460.

50. Wei Z, Spizzo I, Diep H, Drummond GR, Widdop RE, Vinh A. 2014 Differential phenotypes of tissue-infiltrating $\mathrm{T}$ cells during angiotensin II-induced hypertension in mice. PLoS One $\mathbf{9}$, e114895.

51. Itani HA, McMaster Jr WG, Saleh MA, Nazarewicz RR, Mikolajczyk TP, Kaszuba AM, Konior A, Prejbisz A, Januszewicz A, Norlander AE, Chen W, Bonami RH, Marshall AF, Poffenberger G, Weyand CM, Madhur MS, Moore DJ, Harrison DG, Guzik TJ. 2016 Activation of human T cells in hypertension: studies of humanized mice and hypertensive humans. Hypertension $\mathbf{6 8}$, 123-132.

52. Harrison DG, Guzik TJ, Lob HE, Madhur MS, Marvar PJ, Thabet SR, Vinh A, Weyand CM. 2011 Inflammation, immunity, and hypertension. Hypertension 57, 132-140. 
53. Caillon A, Paradis P, Schiffrin EL. 2019 Role of immune cells in hypertension. British journal of pharmacology 176, 1818-1828.

54. Zanoli L, Briet M, Empana JP, Cunha PG, Mäki-Petäjä KM, Protogerou AD, Tedgui A, Touyz RM, Schiffrin EL, Spronck B, Bouchard P, Vlachopoulos C, Bruno RM, Boutouyrie P, Association for Research into Arterial Structure, Physiology (ARTERY) Society, the European Society of Hypertension (ESH) Working Group on Vascular Structure and Function, and the European Network for Noninvasive Investigation of Large Arteries. 2020 Vascular consequences of inflammation: a position statement from the ESH Working Group on Vascular Structure and Function and the ARTERY Society. Journal of Hypertension 38, 1682-1698.

55. Ramachandra AB, Humphrey JD, Marsden AL. 2017 Gradual loading ameliorates maladaptation in computational simulations of vein graft growth and remodelling. Journal of The Royal Society Interface 14, 20160995.

56. Irons L, Humphrey JD. 2020 Cell signaling model for arterial mechanobiology. PLoS computational biology 16, e1008161. 


\begin{tabular}{|c|c|c|}
\hline \multicolumn{3}{|c|}{ Passive response } \\
\hline Diagonal Collagen Orientation & $\alpha_{0}$ & $29.9^{\circ}$ \\
\hline Layer-specific Collagen Fractions & $\beta_{A}^{\theta}, \beta_{M}^{z}=\beta_{A}^{z}$ & $0.056,0.067$ \\
\hline Elastin Parameter & $c^{e}$ & $89.71 \mathrm{kPa}$ \\
\hline Elastin Deposition Stretches & $G_{r}^{e}, G_{\theta}^{e}, G_{z}^{e}$ & $1 / G_{\theta}^{e} G_{z}^{e}, 1.90,1.62$ \\
\hline Muscle Parameters $(o)$ & $c_{1 o}^{m}, c_{2 o}^{m}$ & $261.4 \mathrm{kPa}, 0.24$ \\
\hline Muscle Parameters $(h)$ & $c_{1 h}^{m}, c_{2 h}^{m}$ & $155.7 \mathrm{kPa}, 1.2$ \\
\hline Collagen Parameters $(o)$ & $c_{1 o}^{c}, c_{2 o}^{c}$ & $234.9 \mathrm{kPa}, 4.08$ \\
\hline Collagen Parameters $(h)$ & $c_{1 h}^{c}, c_{2 h}^{c}$ & $27.68 \mathrm{kPa}, 9.98$ \\
\hline Deposition Stretches $(o)$ & $G_{o}^{m}, G_{o}^{c}$ & $1.20,1.25$ \\
\hline Deposition Stretches $(h)$ & $G_{h}^{m}, G_{h}^{c}$ & $1.23,1.21$ \\
\hline \multicolumn{3}{|c|}{ Active response } \\
\hline Smooth Muscle Tone & $T_{\max }$ & $258 \mathrm{kPa}$ \\
\hline Minimal / Maximal Contraction Stretches & $\lambda_{0}, \lambda_{\mathrm{M}}$ & $0.6,1.1$ \\
\hline Vasoactive Parameters & $C_{B}, C_{S}$ & $0.833,1.666$ \\
\hline Rate Parameter & $k_{a c t}$ & $1 / 7$ day $^{-1}$ \\
\hline \multicolumn{3}{|c|}{ Mechano-regulated G\&R } \\
\hline Medial Collagen Gains & $K_{M \sigma}^{c}, K_{M \tau_{w}}^{c}, K_{M \varrho_{\varphi}}^{c}$ & $2.00,2.50,1.74$ \\
\hline Medial Smooth Muscle Gains & $K_{M \sigma}^{m}, K_{M \tau_{w}}^{m}, K_{M \varrho \varphi}^{m}$ & $4 / 5 \times\left[K_{M \sigma}^{c}, K_{M \tau_{w}}^{c}, K_{M \varrho_{\varphi}}^{c}\right]$ \\
\hline Adventitial Collagen Gains & $K_{A \sigma}^{c}, K_{A \tau_{w}}^{c}, K_{A \varrho \varphi}^{c}$ & $5 / 3 \times\left[K_{M \sigma}^{c}, K_{M \tau_{w}}^{c}, K_{M \varrho_{\varphi}}^{c}\right]$ \\
\hline Mass Removal Rates & $k_{M 0}^{m}, k_{M 0}^{c}, k_{A 0}^{c}$ & {$[1 / 7,1 / 7,1 / 7] \mathrm{day}^{-1}$} \\
\hline \multicolumn{3}{|c|}{ Stress-dependent inflammation } \\
\hline Stress Threshold & $\sigma_{v}^{*}$ & $170 \mathrm{kPa}$ \\
\hline Adaptive Response Gain & $K_{\varphi}$ & 2.5 \\
\hline Maladaptive Response Parameters & $\bar{\mu}_{\varphi}^{+}, \bar{\mu}_{\varphi}^{-}$ & $0.102,0.0008$ day $^{-2}$ \\
\hline Rate Parameter & $k_{\varphi}$ & $2 / 7$ day $^{-1}$ \\
\hline
\end{tabular}

Table 1. G\&R model parameters for both an original / basal (subscript $o$; control) and an evolved-to-new homeostatic (subscript $h$; Angll infused) state for the descending thoracic aorta (DTA) from wild-type (C57BL/6) mice [20,31]. "Elastin", "Muscle", and "Collagen" parameters represent elastin-dominated isotropic and smooth muscle / collagendominated anisotropic contributions, with glycosaminoglycans and other constituents not specified explicitly. Subscripts $M$ and $A$ refer to medial and adventitial respectively. Smooth muscle and collagen parameters and deposition stretches evolve from normotensive (adaptive, $o$ ) to hypertensive (maladaptive, $h$ ) conditions with the extent of the inflammatory cell fraction $\Delta \varrho_{\varphi} \in[0,1]$ as $c_{1,2}^{m, c}\left(\Delta \varrho_{\varphi}\right)=c_{1,2 \mid o}^{m, c}+\left(\Delta \varrho_{\varphi}\right)^{1 / 3}\left(c_{1,2 \mid h}^{m, c}-c_{1,2 \mid o}^{m, c}\right)$ and $G^{m, c}\left(\Delta \varrho_{\varphi}\right)=$ $G_{o}^{m, c}+\left(\Delta \varrho_{\varphi}\right)^{1 / 3}\left(G_{h}^{m, c}-G_{o}^{m, c}\right)$. 

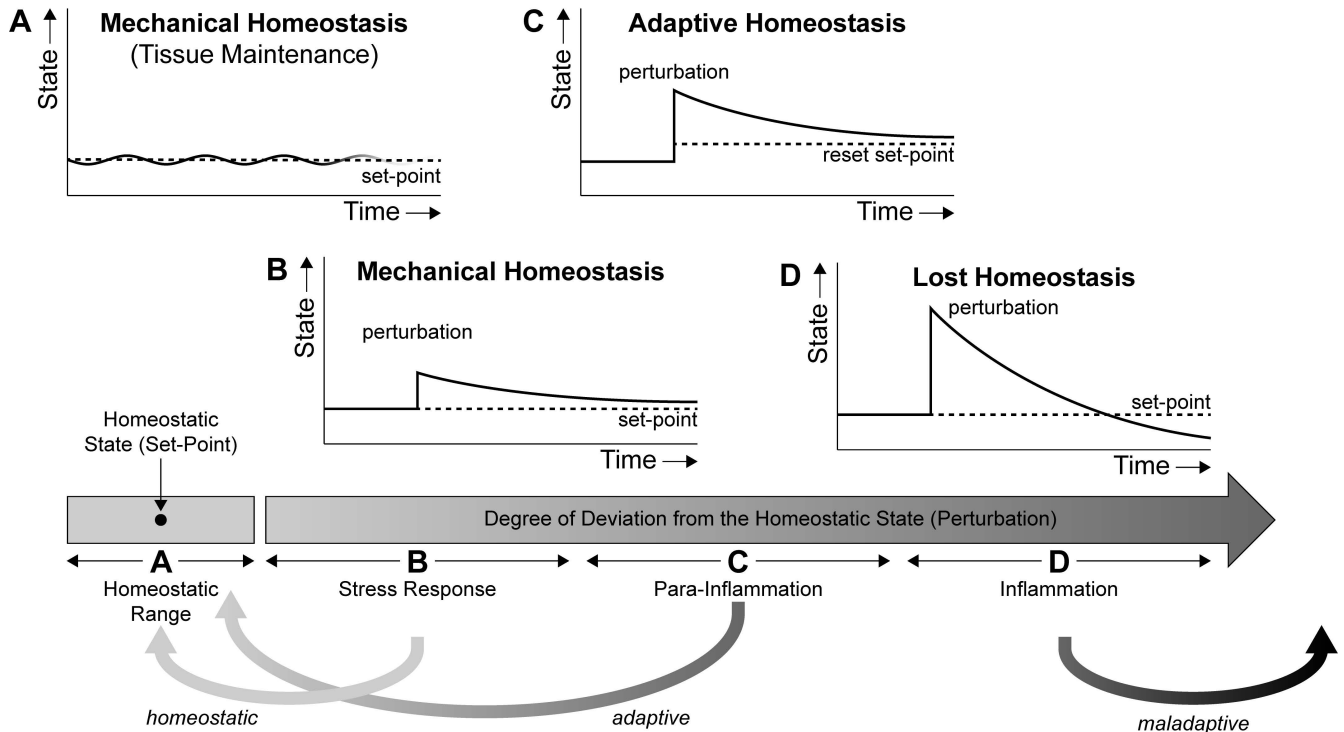

Figure 1. Schema of complementary or contrasting roles of mechano- and immuno-regulation in tissue homeostasis, with potential lost homeostasis in extreme cases. In particular, (A) mechanical homeostasis maintains near normal tissuelevel composition, structure, properties, and function over long periods in health despite continual turnover of many intramural constituents during transient changes in hemodynamics on a daily basis; (B) mechanical homeostasis can restore mechano-regulated variables (i.e., states) toward normal following marked perturbations, as, for example, modest sustained changes in hemodynamics; (C) mechanical and inflammatory processes can work together to restore regulated variables toward normal, though at times with a resetting of set-points; (D) severe or sustained (chronic) inflammation can over-ride normal homeostatic processes, thus resulting in maladaptation or additional disease progression, as, for example, in highly fibrotic responses in some vessels during hypertension. Although particular responses (A-D) are suggested to define particular ranges, these responses are expected to represent a continuous spectrum. Overall schema inspired by [13], though presented in a different context.

ज

政

列

$\frac{1}{0}$

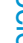

: 
A

Stimulus function for production

Stored energy function for of constituent $\alpha$ in layer $\Gamma$ :
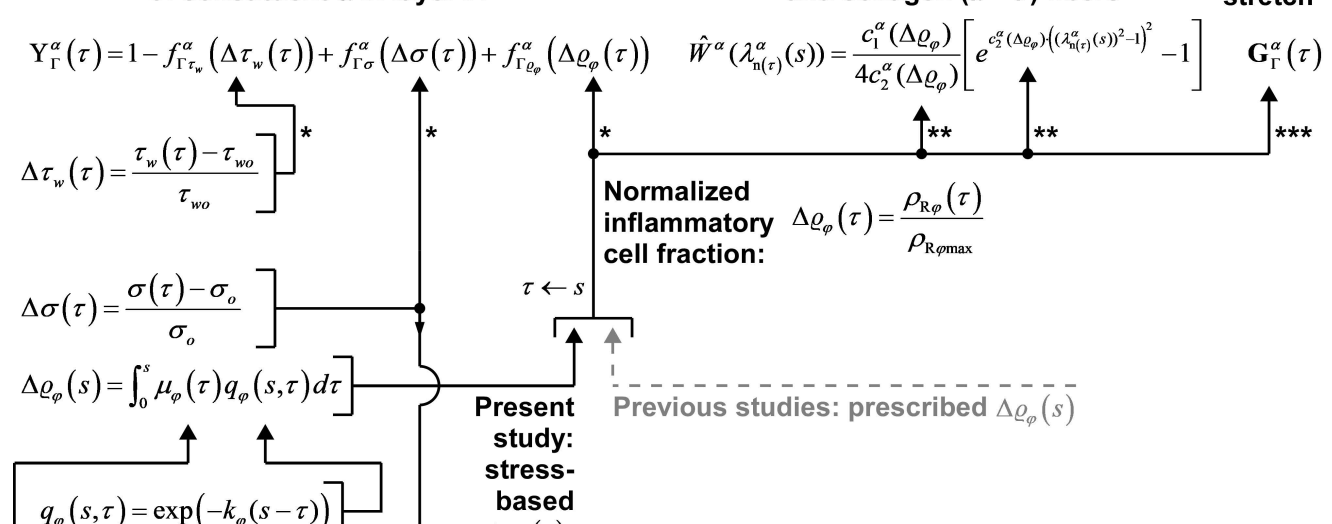

$$
\begin{aligned}
& \left.q_{\varphi}(s, \tau)=\exp \left(-k_{\varphi}(s-\tau)\right)\right] \quad \begin{array}{c}
\text { based } \\
\Delta \varrho_{\varphi}(s)
\end{array} \\
& {\left[\begin{array}{lc}
\mu_{\varphi}(\tau)=k_{\varphi} K_{\varphi}\langle\Delta \sigma(\tau)\rangle & \text { if } \sigma(\bar{\tau})<\sigma^{*} \\
\frac{d \mu_{\varphi}(\tau)}{d \tau}=\bar{\mu}_{\varphi}^{+} & \forall \bar{\tau} \leq \tau \\
\frac{d \mu_{\varphi}(\tau)}{d \tau}=-\bar{\mu}_{\varphi}^{-} & \text {if } \sigma(\tau) \geq \sigma^{*} \\
& \text { if } \sigma(\tau)<\sigma^{*} \text { and } \\
& \exists \bar{\tau}<\tau \mid \sigma(\bar{\tau}) \geq \sigma^{*}
\end{array}\right.}
\end{aligned}
$$

Legend:

Adaptive inflammatory remodeling

Maladaptive inflammatory remodeling

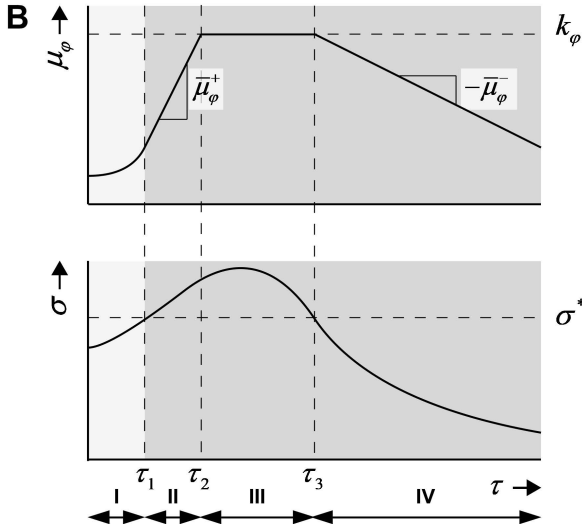

Figure 2. A. Schema of how inflammation influences the turnover of smooth muscle cells and collagen fibers via their stimulus functions for mass density production, passive mechanical properties, and deposition stretches. B. Typical time course of $\mu_{\varphi}$ and $\sigma$ progression. In period I, stress $(\sigma)$ is below its threshold $\sigma^{*}$, and inflammation contributes to homeostasis. At time $\tau_{1}$, stress passes its threshold and $\mu_{\varphi}$ starts increasing linearly at a rate $\bar{\mu}_{\varphi}^{+}$(period II), until it saturates at a value $k_{\varphi}$ at time $\tau_{2}$. As long as $\sigma \geq \sigma^{*}, \mu_{\varphi}$ remains saturated at a level of $k_{\varphi}$ (period III). As soon as $\sigma$ drops below $\sigma^{*}$ at time $\tau_{3}, \mu_{\varphi}$ starts decreasing linearly at a rate of $-\bar{\mu}_{\varphi}^{-}$(period IV). If, in phase II, $\sigma$ drops below $\sigma^{*}$ before $\mu_{\varphi}$ reaches $k_{\varphi}$, no plateau in $\mu_{\varphi}$ (no phase III) occurs. See Table 1 or [20] for specific functional dependencies $\left(*,{ }^{* *},{ }^{* *}\right)$ used in the present study. 

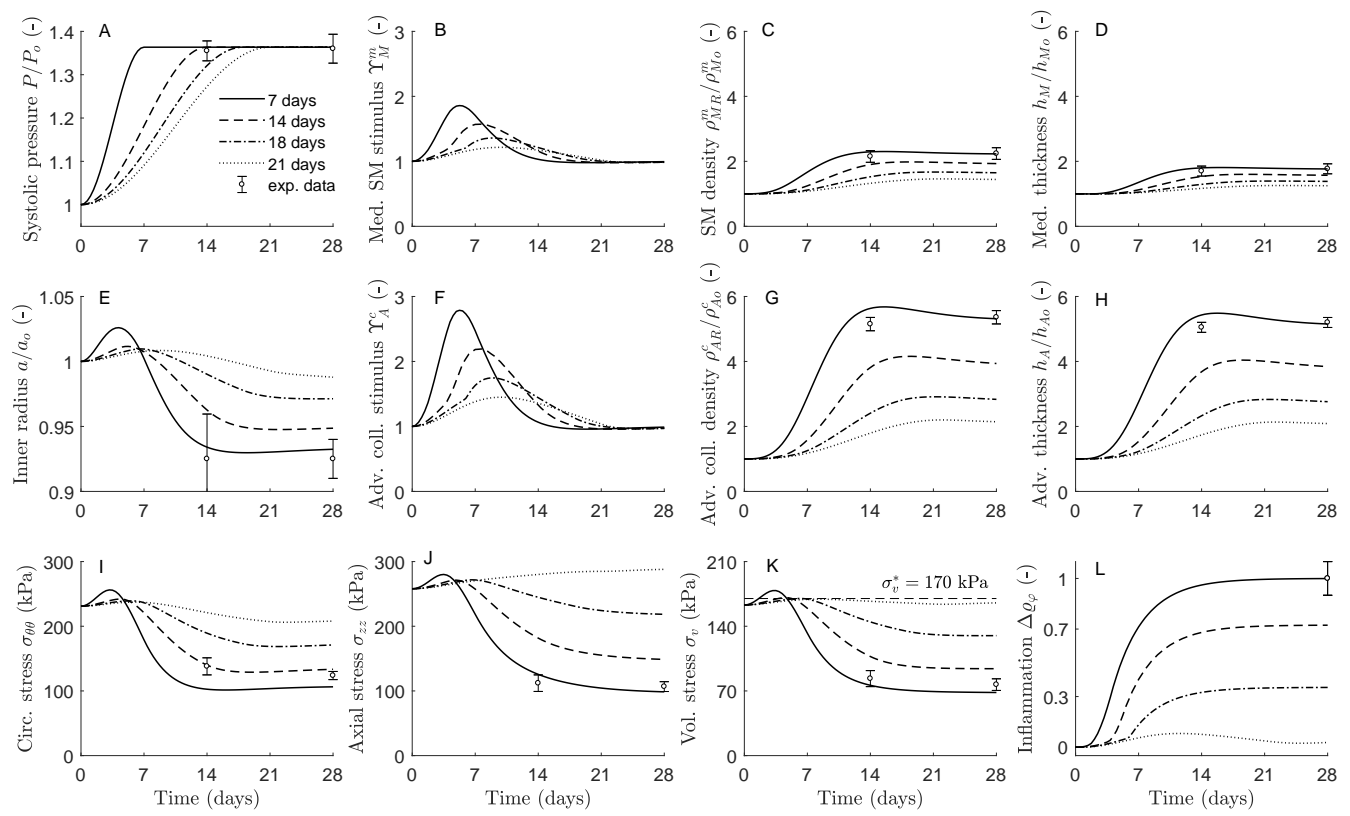

Figure 3. Different hypertensive remodeling responses predicted by the present coupled stress-driven inflammation G\&R model for different rates of increase in blood pressure over 1 (solid lines) to 3 (dotted lines) weeks, yielding progressively more adaptive remodeling responses. Shown, too, are mean \pm SEM experimental values (open circles with error bars) extracted from [31], which reports remodeling at two and four weeks given a rapid increase in pressure within about one week. Note that the temporal profile for pressure $P$ is the only input to the model; all other variables, including the extent of inflammation $\Delta \varrho_{\varphi}$, are computed (i.e., predicted) as part of the solution of the coupled model. The predictions match the available experimental data well. Albeit not shown, the in vivo value of axial stretch $\sim 1.7$ (normotensive, $s=0$ ) was predicted to decrease with hypertensive remodeling in all cases; in particular to $\sim 1.35$ (fully maladaptive, solid line) or $\sim 1.6$ (adaptive, dotted line), consistent with both experimental findings and prior predictions in [20] with prescribed inflammation. Note for the latter that a slight over-thickening (relative to an ideal mechanoadaptation [29]) combined with the recovery of the target stress / set-point (i.e., with absent overriding inflammatory effects) to yield a decrease in circumferential stress and associated increase in axial stress (hence an increase in axial force required to maintain the, herein assumed fixed, in vivo axial length; not shown). There remains a pressing need for more data on axial behavior, which are not available during in vivo studies, in order to identify an ideal stimulus function that accounts for differential circumferential and axial remodeling. 

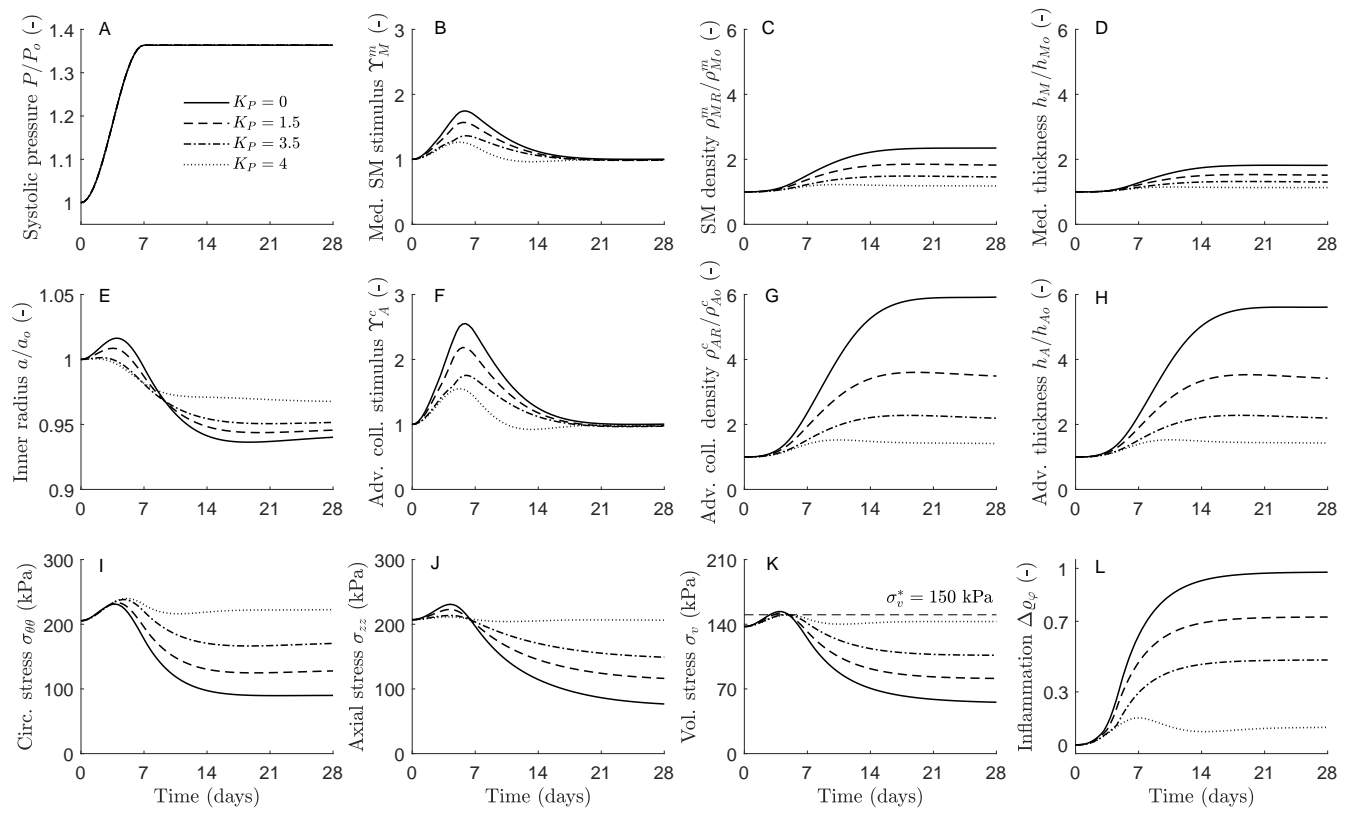

Figure 4. Different hypertensive remodeling responses predicted by the coupled stress-driven inflammation G\&R model for gradual increases in contractile tone $T_{\max }(s)=T_{\max }(0)\left(1+K_{P}(P(s)-P(0)) / P(0)\right)$ in Eq. (2.12) from $K_{P}=$ 0 (constant tone, solid lines) to $K_{P}=4$ (maximally enhanced tone with hypertension, dotted lines), yielding progressively more adaptive remodeling responses. Note that the temporal profile for pressure $P$ is the only input to the model; all other variables, including the extent of inflammation $\Delta \varrho_{\varphi}$, are computed as part of the solution of the coupled model. There are no data available to which to compare. 

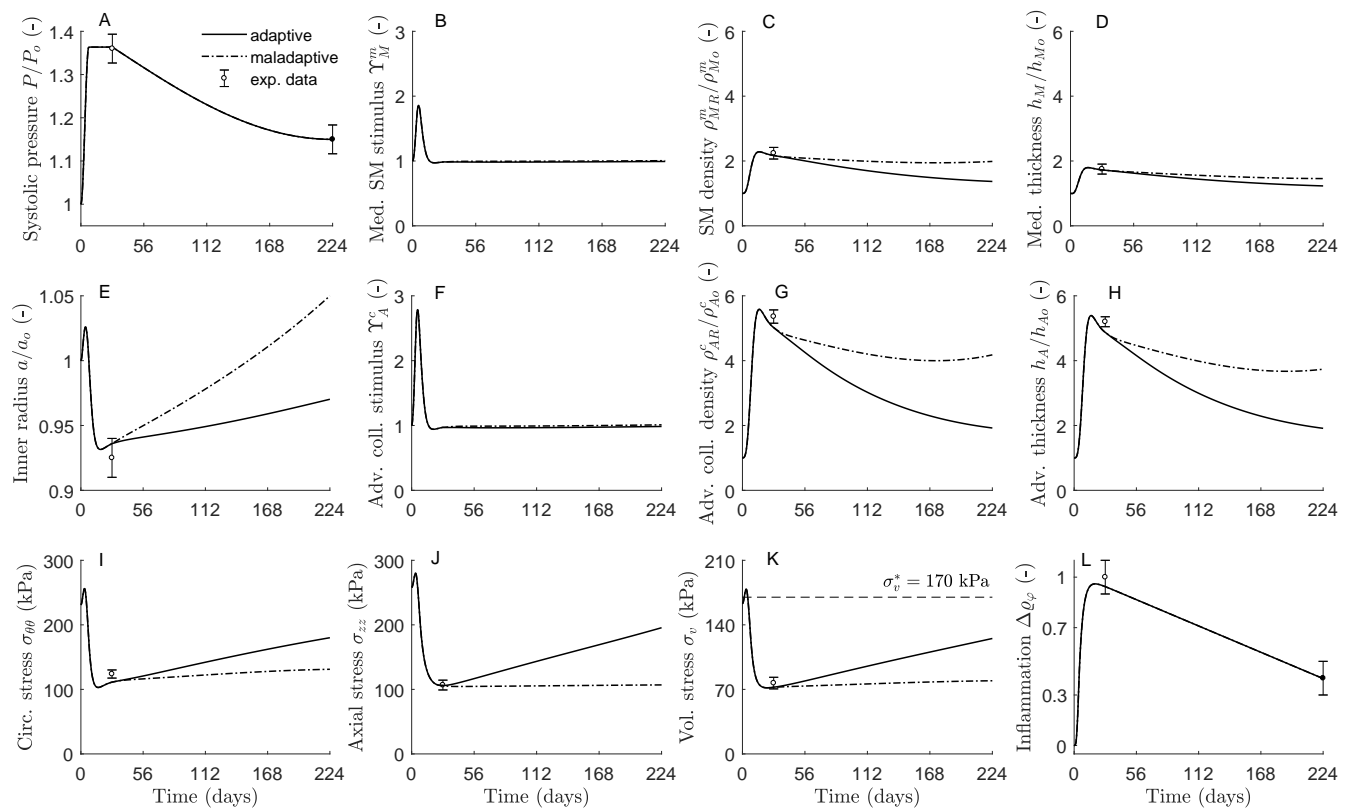

Figure 5. Model predictions of long-term responses up to 196 days after terminating the Angll infusion at 28 days following a 1.36-fold increase in systolic pressure (A, model input) that persists up to $\mathrm{s}=28$ days but drops to $P / P_{o}=1.15$ during the subsequent 196 days. Predictions are shown with (solid line) and without (dash-dotted) recovery of passive properties of smooth muscle cells and collagen fibers toward their original homeostatic values with the computed (partial) reversal of inflammation (L). Shown, too, are mean \pm SEM experimental values (open circles with error bars) extracted from [31] at 28 days and (pre-)assumed values (solid circles with error bars) for the remnant pressure and inflammation at 224 days consistent with [22] and [21]. There is a pressing need for more longitudinal data on potential recovery of vascular properties following elimination of a prior long-term perturbation. 